\title{
Large Eddy Simulation of Premixed Turbulent Flames using the Probability Density Function Approach
}

\author{
I. A. Dodoulas and S. Navarro-Martinez \\ Department of Mechanical Engineering, Imperial College London, \\ Exhibition Road, London SW7 2AZ,UK \\ email: s.navarro@imperial.ac.uk. \\ Tel: 44-20-75949229
}

\begin{abstract}
In the present study a Large Eddy Simulation and Filtered Density Function model is applied to three premixed piloted turbulent methane flames at different Reynolds Numbers using the Eulerian stochastic fields approach. The model is able to reproduce the flame structure and flow characteristics with a low number of fields (between 4 and 16 fields). The results show a good agreement with experimental data with the same closures employed in non-premixed combustion without any adjustment for combustion regime. The effect of heat release on the flow field is captured correctly. A wide range of sensitivity studies is carried out, including the number of fields, the chemical mechanism, differential diffusion effects and micro-mixing closures. The present work shows that premixed combustion (at least in the conditions under study) can be modelled using LES-PDF methods.. Finally, the ability of the model to predict flame quenching is studied. The model can accurate capture the conditions at which combustion is not sustainable and large pockets of extinction appear.
\end{abstract}

Key words: LES, PDF methods, turbulent combustion, premixed flames.

\section{Introduction}

Large Eddy Simulation (LES) and Probability Density Function (PDF) have obtained recent success in simulating non-premixed and partially premixed turbulent flames [1-5] among others, following both the Lagrangian and the Eulerian Approaches. The PDF framework in principle offers the advantage of being combustion-regime independent as no assumptions are made in its derivation. However, its application to premixed flows has been restricted and its applicability there questioned [6], due in part to the need in RANSPDF approaches to modify the micro-scale mixing to include a molecular length scale and the need to resolve the steep gradients in the reactive scalars.

Direct application of RANS combustion models to LES simulations poses significant difficulties in premixed flames. The flame front is in most applications much thinner than the filter width used typically in LES simulations. As a consequence, large sub-grid temperature fluctuations are created in a relatively small number of cells and the flame front cannot be typically resolved directly. The propagation speed of the flame front is intimately linked to the number of grid points across it and large errors occur if the flame is under-resolved. There are several approaches in the literature to premixed modelling in the LES context. They can broadly be divided into two main approaches [7]: one, where a solution for a progress-type variable is sought and then the flame structure is recovered and another where the reactive scalars are solved and the progress variable and flame position are derived from the solution. The first attempts to use LES in premixed flames extended the RANS ideas of the Eddy-Break-Up model (EBU) to LES [8]. However, they 
did not address the problem of under-resolution of the flame front. More advanced methods include the artificially thickened flame (ATF/TFM) [9], where the chemical source term is modified to allow the flame to "thicken" and become larger than the mesh size; and the G-equation $[10,11]$, where the flame front is explicitly tracked. All these methods require accurate predictions for the turbulent flame speed, which lead to the development of the Flame Surface Density (FSD) approaches [12,13], the Flame Surface Wrinkling Model [14] and the Coherent Flame Model reformulated for LES [15]. All these methods have common characteristics as they all look for a closure of the turbulent flame speed through the FSD equation and they are restricted to the premixed regime, in most cases away from the broken reaction zones. Other premixed-specific approaches found in the literature include variants of the flamelet concept typical of non-premixed flames; the flamelet generated manifold method (FGM) [16], application of the similarity model [17] and the broadened flame model [18] among others. A complete review of LES models for premixed combustion is outside the scope of this paper, and the reader is referred to the literature [19]. The second tier of combustion modelling approaches uses formulations that can be in principle applied to both premixed and non-premixed regimes, the most popular among these are the Linear Eddy Model [20] and the PDF (discussed further below). Recent trends are to combine several combustion methods to generalize the approach to both combustion regimes. Examples are presumed-PDF/FSD [21] Presumed Conditional Moments/FPI [22] and ATF/FGM [23]

Despite its success in other combustion regimes, the application of PDF methods to turbulent premixed flames has been limited. Studies in the RANS-PDF context have revealed that modifications to the micro-mixing term have to be taken into account for accurate predictions [24]. Numerous other RANS-PDF studies also suggest that the micro-mixing model plays a very important role and conventional closures can lead to relatively inaccurate results [25-28]. References [30] and [31] applied the LES-PDF methodology (using different formulations) successfully to a premixed flame using conventional closures derived from non-premixed combustion. It can be argued that in LES-PDF the improvement of large scale mixing allows the use of conventional closures that reproduce well high Reynolds turbulent flames.

In the present work a comprehensive study of the application of the LES-PDF method to three different premixed flames is performed. The objective is to assess the validity of the approach at different Reynolds and Damkhöler numbers and quantify the importance of the different contributions. This paper is organized as follows: First, a brief description of the LES-PDF method and the Eulerian Stochastic Field approach is presented, together with discussion of the assumptions employed. Afterwards, the test case of the turbulent premixed flames is described (experimental set-up as well as numerical implementation). The paper continues with a discussion of the effects of several parameters on the simulation results and examines the suitability of the LES-PDF method to describe several premixed flame regimes. 


\section{Modelling}

\subsection{The Filtered Navier-Stokes Equations}

In LES a spatial filter is applied to the equations of motion: the spatial filter of a function $f \equiv f(\mathbf{x}, t)$ is defined as its convolution with a filter function, $G$, according to:

$$
\bar{f}(\mathbf{x}, t)=\int_{V} G\left(\mathbf{x}-\mathbf{x}^{\prime}, \Delta\right) f\left(\mathbf{x}^{\prime}, t\right) d V
$$

Where the filter function must be positive definite and have a characteristic width of $\Delta$ which, in general, may vary with the position. More particularly, the filter width is taken as the cubic root of the local grid cell volume. The density variations in the unresolved scales that arise in combusting flows can be treated through the use of density weighted, or Favre, filtering, defined by:

$$
\tilde{f}=\frac{\overline{\rho f}}{\bar{\rho}}
$$

Application of the density weighted filtering operation results in the filtered equations of motion (continuity, momentum, $N_{S}$ chemical species transport and energy - enthalpy equations in this order):

$$
\begin{gathered}
\frac{\partial \bar{\rho}}{\partial t}+\frac{\partial \bar{\rho} \tilde{u}_{j}}{\partial x_{j}}=0 \\
\frac{\partial \bar{\rho} \tilde{u}_{i}}{\partial t}+\frac{\partial \bar{\rho} \tilde{u}_{j} \tilde{u}_{i}}{\partial x_{j}}=-\frac{\partial \bar{p}}{\partial x_{i}}+\frac{\partial \bar{\tau}_{i j}}{\partial x_{j}}-\frac{\partial \tau_{i j}^{s g s}}{\partial x_{j}} \\
\frac{\partial \bar{\rho} \tilde{Y}_{k}}{\partial t}+\frac{\partial \bar{\rho} \tilde{u}_{j} \tilde{Y}_{k}}{\partial x_{j}}=\frac{\partial \bar{J}_{j}^{k}}{\partial x_{j}}-\frac{\partial J_{j}^{k, s g s}}{\partial x_{j}}+\widetilde{\rho} \widetilde{\dot{\omega}_{k}} \quad k=1, \ldots, N_{S} \\
\frac{\partial \bar{\rho} \tilde{h}}{\partial t}+\frac{\partial \bar{\rho} \tilde{u}_{j} \tilde{h}}{\partial x_{j}}=\frac{\partial \bar{J}_{j}^{h}}{\partial x_{j}}-\frac{\partial J_{j}^{h, s g s}}{\partial x_{j}}-\sum_{k} h_{k} \frac{\partial}{\partial x_{j}}\left[\left(D-D_{k}\right) \frac{\partial \bar{\rho} \tilde{Y}_{k}}{\partial x_{j}}\right]+\frac{\overline{D p}}{D t}+\Phi_{v}
\end{gathered}
$$

where the symbols have their usual meaning. Fick's diffusion is used for the species diffusion, $J_{j}$, where Dufour and Soret effects are neglected. In the case of differential diffusion, a correction velocity is introduced in (5) to guarantee species mass conservation together with Hischfelder and Curtiss approximation [7]. If differential diffusion is not taken into account, then the correction velocity term is dropped. In the enthalpy equation, at low Mach number open flames it is common to neglect the pressure variations and the viscous friction, the last two terms in the RHS of Eqn. (6).

Equations (4-6) need closure for the sub-grid contribution. In the momentum equation, the deviatoric part of the sub-grid scale stress tensor $\tau_{i j}^{s g s}=\bar{\rho}\left(\widetilde{u_{i} u_{j}}-\tilde{u}_{i} \tilde{u}_{j}\right)$ is determined with the standard Smagorinsky model, in which the anisotropic sub-grid stresses are assumed proportional to the filtered strain rate $\tilde{S}_{i j}$. The proportionality constant has the 
dimensions of viscosity and is referred to as turbulent (or sub-grid) viscosity $\mu_{s g s}$. The model is given by the following equation[2,3,30,32]:

$$
\mu_{s g s}=\bar{\rho}\left(C_{S} \Delta^{2}\right)\left\|\tilde{S}_{i j}\right\|
$$

Where $C_{S}$ is the Smagorinsky constant and $\left\|\tilde{S}_{i j}\right\|=\sqrt{2 S_{i j} S_{i j}}$ is the Frobenius norm of the resolved strain tensor. The value of $C_{S}$ can largely vary between homogenous regions and the vicinity of a wall [32], nevertheless in the present work, a universal value of $C_{S}=0.09$ is adopted [33].

At low Mach numbers, the reactive species mass fractions and enthalpy can be expressed as $N_{S}+1$ (number of species considered plus enthalpy) convection-diffusion-reaction equations with identical molecular diffusion coefficient $D=D_{k}$. The next section describes the equations to obtain the filtered moments, $\tilde{Y}_{k}$, of these through the solution of the Filtered Probability Density Function.

\subsection{Filtered Probability Density Function}

Using the filtering operation as stated in Eqn. (1), a density-weighted sub-grid (or filtered) PDF for the $N_{S}+1$ scalar quantities can be defined as follows [34]:

$$
\bar{\rho} \tilde{P}(\mathbf{x}, t ; \underline{\psi})=\int_{V} \rho \prod_{k=1}^{N_{S}} \delta\left(\psi_{k}-Y_{k}(\mathbf{x}, t) G\left(\mathbf{x}-\mathbf{x}^{\prime} ; \Delta\right) d V^{\prime}\right.
$$

where $\psi$ represents the sample space of the $k$-th reactive species. A transport equation for the filtered PDF can be derived from the appropriate conservation equations, leading to:

$$
\begin{aligned}
& \frac{\partial \bar{\rho} \tilde{P}}{\partial t}+\frac{\partial \bar{\rho} \tilde{u}_{j} \tilde{P}}{\partial x_{j}}-\frac{\partial}{\partial x_{j}}\left[\bar{\rho} D_{e} \frac{\partial \tilde{P}}{\partial x_{j}}\right]= \\
& -\sum_{\alpha=1}^{N_{S}} \frac{\partial}{\partial \psi_{\alpha}}\left[\rho \dot{\omega}_{\alpha}(\underline{\psi}) \tilde{P}\right]-\sum_{\alpha=1}^{N_{S}} \frac{\partial^{2}}{\partial \psi_{\alpha} \psi_{\beta}}\left[\bar{\rho} D \overline{\frac{\partial Y_{\alpha}}{\partial x_{j}} \frac{\partial Y_{\beta}}{\partial x_{j}} \mid \underline{Y}=\underline{\psi} \tilde{P}}\right]
\end{aligned}
$$

In the above equation, the spatial, temporal and scalar dependencies have been dropped for compactness. A gradient diffusion type approximation [35] has been applied to model the PDF transport by sub-grid turbulent fluctuations. The combined molecular diffusion and turbulent transport coefficient is given by an effective diffusion coefficient $D_{e}$ [36] where $D_{e}=D+D_{s g s}=\left(\mu / S c+\mu_{s g s} / S c_{s g s}\right) / \bar{\rho}$ and $S c_{s g s}$ is a constant sub-grid Schmidt number assigned the value 0.7 [37]. In the case of differential diffusion, an additional term would need to be added in the right hand side of (9) to include additional transport in spatial and scalar spaces. The final term of the PDF transport equation (9) is called the micromixing or sub-grid scale mixing and represents the effect of molecular diffusion on the PDF. In this work the Linear Mean Square Estimation (LMSE) closure is used [38] also known as Interaction by Exchange with the Mean [39], thus the final term in (9) is replaced by: 


$$
\sum_{\alpha=1}^{N_{S}} \frac{\partial^{2}}{\partial \psi_{\alpha} \psi_{\beta}}\left[\bar{\rho} D \overline{\frac{\partial Y_{\alpha}}{\partial x_{j}} \frac{\partial Y_{\beta}}{\partial x_{j}} \mid \underline{Y}=\underline{\psi} \tilde{P}}\right] \approx \frac{\bar{\rho}}{T_{s g s}} \sum_{\alpha=1}^{N_{S}} \frac{\partial}{\partial \psi_{\alpha}}\left[\left(\psi_{\alpha}-\tilde{Y}_{\alpha}\right) \tilde{P}\right]
$$

The sub-grid mixing time scale $T_{s g s}$ is assumed proportional to the velocity time scale:

$$
\frac{1}{T_{s g s}}=C_{\Phi}\left(\frac{\mu+\mu_{s g s}}{\bar{\rho} \Delta^{2}}\right)
$$

The above term decreases the variance of the sub-grid PDF and in the limit of infinite micromixing the sub-grid PDF relaxes towards a delta Dirac. The above model would recover the Dirac delta in the DNS limiting case of $\Delta \rightarrow 0$ [36]. The above time scale includes a molecular time scale, proportional to the diffusion coefficient (through $\mu$ ), this fact combined with the molecular diffusion present in $D_{e}$ makes LES-PDF suitable for premix calculations. There are several alternative micromixing methods proposed mainly in the RANS context $[40,41]$ however the increase in complexity and computational time required does not necessary guarantee improved predictions in LES context.

It has been argued that in RANS applications to inert flows [42], a constant of $C_{\Phi}=2$ often provides the correct scalar dissipation rate for a passive scalar in equilibrium flows. However, this constant cannot be considered universal and experimental studies of temperature decay in grid-generated turbulence [43] have shown a variation of $C_{\Phi}$ between 0.67 and 2.38. In LES the energetic motions are resolved and the representation of the sub-grid scale mixing provided by the LMSE model may be adequate for many practical situations $[4,36]$

In premixed flames, large instantaneous sub-grid variances can exist even in low Reynolds numbers flames (as the flame front is often much smaller than all fluid scales). A scaling of the micro-mixing time scale was proposed in partially premixed and lifted flames [42] to increase the mixing in such regions, ensuring that micro-mixing tends towards infinity if the flow is fully resolved and becomes effective if the turbulent viscosity is small compared to the molecular viscosity or is of the same order. The constant is then redefined as:

$$
C_{\Phi}=C_{\Phi}^{0}\left(1+\frac{\mu}{\mu_{s g s}}\right)
$$

where $\mu_{s g s} / \mu \propto \operatorname{Re}$. The above equation decays to the constant value $C_{\Phi}^{0}$ when the subgrid viscosity $\mu_{s g s} \neq 0$. In the laminar case, $\operatorname{Re} \rightarrow 0$ and $C_{\Phi}^{0} \rightarrow \infty$, and the sub-grid PDF becomes a delta Dirac. The micromixing constant is then taken as $C_{\Phi}^{0}=2$, its effect on the predictions together with the scaling will be investigated in detail in section 4.4. 


\subsection{Stochastic Field Method}

This section describes the Stochastic Field Formulation and its current implementation. The solution method is a fully Eulerian method in which the continuous fields represent an equivalent stochastic system with the same one-point PDF Equation as Eqn (8). Examples of the LES implementation of the stochastic fields can be found in nonpremixed flames [1,5,44], lifted flames [2], spark-ignition [45,83] and spray flames $[46,47]$.

The density weighted sub-grid PDF is represented by an ensemble of stochastic fields $\xi_{k}^{n}(\mathbf{x}, t)$ such that:

$$
\tilde{P}(x, t ; \underline{\psi})=\frac{1}{N} \sum_{n=1}^{N} \prod_{k=1}^{N_{S}} \delta\left[\psi_{k}-\xi_{k}^{n}(\mathbf{x}, t)\right]
$$

The stochastic fields are not any particular realisation of the scalar field, but an equivalent stochastic system (both sets have the same one-point PDF). A system of stochastic partial differential equations (SPDE's) for the stochastic fields can be derived which is equivalent to the PDF evolution equation (9). In general, two approaches can be adopted to derive the SPDE for the stochastic fields: the Ito interpretation [48] and the Stratonovich interpretation [49] of the stochastic integral.

In this paper the Ito interpretation is followed and the corresponding SPDE for the stochastic field is

$$
\bar{\rho} \frac{d \xi_{k}^{n}}{d t}+\bar{\rho}_{\tilde{u}_{j}} \frac{\partial \xi_{k}^{n}}{\partial x_{j}}=\frac{\partial}{\partial x_{j}}\left[\bar{\rho} D_{e} \frac{\partial \xi_{k}^{n}}{\partial x_{j}}\right]=\bar{\rho} \dot{\omega}_{k}(\underline{\xi})-\frac{\bar{\rho}}{T_{s g s}}\left(\xi_{k}^{n}-\tilde{Y}_{k}\right)+\bar{\rho} \sqrt{2 D_{e}} \frac{\partial \xi_{k}^{n}}{\partial x_{j}} \frac{d W_{j}^{n}}{d t}
$$

The last term of this equation includes, $d \mathbf{W}^{\mathrm{n}}$, which represents increments of a Wiener process with 0 mean and variance $d t$. The stochastic term is different for each stochastic field and independent of the spatial location. The stochastic fields in (13) are smooth on the scale of the filter width, continuous and differentiable in space and continuous but not differentiable in time. In the above equation, the last term (which includes the increments of the Wiener process) increases the width of the PDF, while the micromixing term opposes it. If the sum of the last two terms in (14) is 0 , the stochastic fields behave like identical sets of reactive scalars following Eq. (5). The Equation (14) preserves the boundedness of the scalar as the gradient of the fields vanish when the scalars go to extrema and therefore the stochastic contribution vanish. Each field satisfies the mass conservation and bound properties of the modelled PDF equation; this means that the species mass fractions will remain positive and sum to unity. In the Ito interpretation the filtered value of the stochastic term vanishes for large number of fields:

$$
\left\langle\sqrt{2 D_{e}} \frac{\partial \xi_{k}^{n}}{\partial x_{j}} \frac{d W_{j}^{n}}{d t}\right\rangle \rightarrow 0
$$

The Favre filtered values of a $k$-scalar are simply obtained by ensemble averaging the stochastic fields 


$$
\tilde{Y}_{k}=\frac{1}{N} \sum_{n=1}^{N} \xi_{k}^{n}
$$

It can be argued [36] that the Stratonovich interpretation may be more appropriate to solve discontinuities, as the fields do not have to be continuous in space and the equivalent Stratonovich SPDE is hyperbolic in nature; allowing for discontinuous solutions as the information travels through stochastic characteristics. However, the flame front does not arise by the hyperbolic part of the reactive equations but from the diffusive-reaction balance and the apparent discontinuity is only due to under-resolution (unlike shock waves, that arise from the Euler equations) and advantages of either formulation are not clear. Applications of stochastic field to premixed flames are rare $[50,79]$ and therefore a judicious choice of stochastic integration scheme cannot be made a priori.

\subsection{Numerical Implementation}

In the present work, the LES equations are solved for the flow and reactive scalar fields using the in-house code BOFFIN [51] in cylindrical coordinates. It is based on a fully implicit low Mach number formulation using a staggered arrangement. Central differences have been used in the momentum equations. The flow solver is then marched in time using a Crank-Nicholson scheme. For the reactive scalars, the spatial gradient of the stochastic term is discretized using central differences. In order to preserve the physical limits of the fields, the advection term is solved using a Total Variation Diminish scheme (TVD), as is common practice in LES to transport bounded scalar and avoid non-physical overshoots [32]. The Wiener process (or random walk) is approximated by time-step increments where $d W_{j}^{k}=\sqrt{d t}\{-1,1\}$, using a dichotomic random vector [52]. This procedure ensures that the random term is bounded and allows the use of fully implicit weak approximations. Moreover, using a Gaussian random number generator for a low number of samples is very inaccurate and results in large errors in mean and variance. The resultant scheme is a weak first order approximation to the SPDE [53].

\section{Test Case}

In this work, the three piloted turbulent premixed Bunsen flames studied by Chen et al. [54] are investigated. The most stretched flame, denoted F1, is located at the borderline to the well stirred reactor regime while the less stretched F3 flame is located in the thin reaction zone regime, close to the borderline of the flamelet regime [54]. This set of flames has been widely studied in the literature, and RANS simulations of all F1-F3 flames have been reported [24,25,55-60], however to the authors' knowledge only three LES simulations have been reported [11,31,82] and mostly on the lower Reynolds number flame F3.

Table 1. Global operating characteristics of the three flames [54]. The Reynolds number value is calculated on the basis of the nozzle diameter and bulk velocity $U_{0} . k_{0}$ denotes the centreline turbulent kinetic energy at the nozzle exit.

\begin{tabular}{|c|c|c|c|}
\hline Flame & F1 & F2 & F3 \\
\hline$U_{0}(\mathrm{~m} / \mathrm{sec})$ & 65 & 50 & 30 \\
\hline
\end{tabular}




\begin{tabular}{|c|c|c|c|}
\hline Reynolds number & 52500 & 40300 & 24200 \\
\hline$k_{0}\left(\mathrm{~m}^{2} / \mathrm{sec}^{2}\right)$ & 12.7 & 10.8 & 3.82 \\
\hline
\end{tabular}

The three flames are generated with the same burner. Table 1 presents the mean nozzle exit velocities, the corresponding Reynolds numbers, and the centreline turbulent kinetic energy values, as provided by experimental measurements [54]. The burner design is shown schematically in Figure 1.



Fig 1 The Premixed Flame Burner Configuration [54]

The burner consists of a nozzle with diameter $D=12 \mathrm{~mm}$ for the main stream (jet stream) which is surrounded by a large pilot stream to stabilize the turbulent main jet flame. The laminar pilot stream is generated by an array (1165 holes of diameter $1 \mathrm{~mm})$ of small jets issued through a cooled perforated plate [54]. In this study, the pilot velocity is estimated as $U_{\text {pilot }}=1.32 \mathrm{~m} / \mathrm{s}$, as followed in RANS-PDF approaches [24]. Both streams consist of a stoichiometric methane-air mixture (the jet stream consists of un-burnt methane and air while the pilot is composed by the products of stoichiometric combustion of the methaneair mixture). Compositions are given in Table 2

Table 2. Jet and Pilot Stream Mass Fraction Composition [24].

\begin{tabular}{|c|c|c|}
\hline Species & Jet & Pilot \\
\hline $\mathrm{Y}_{\mathrm{CH} 4}$ & 0.0552 & - \\
\hline $\mathrm{Y}_{\mathrm{O} 2}$ & 0.2201 & $5.00 \mathrm{E}-04$ \\
\hline $\mathrm{Y}_{\mathrm{H} 2 \mathrm{O}}$ & - & 0.1236 \\
\hline $\mathrm{Y}_{\mathrm{CO} 2}$ & - & 0.15 \\
\hline
\end{tabular}




\begin{tabular}{|c|c|c|}
\hline $\mathrm{Y}_{\mathrm{CO}}$ & - & $7.80 \mathrm{E}-04$ \\
\hline $\mathrm{Y}_{\mathrm{H} 2}$ & - & $3.00 \mathrm{E}-05$ \\
\hline $\mathrm{Y}_{\mathrm{OH}}$ & - & $1.20 \mathrm{E}-04$ \\
\hline $\mathrm{Y}_{\mathrm{N} 2}$ & 0.7247 & 0.72497 \\
\hline
\end{tabular}

The burner is surrounded by air in the form of an external co-flow at $U_{\text {air }}=0.22 \mathrm{~m} / \mathrm{s}$ with $T_{a i r}=298 \mathrm{~K}$. The experimental database includes radial profiles of the mean velocity, the turbulent kinetic energy, mean and variance of the temperature, and the mean mass fractions of $\mathrm{CH}_{4}, \mathrm{CO}_{2}, \mathrm{CO}, \mathrm{O}_{2}, \mathrm{OH}$ and $\mathrm{H}_{2} \mathrm{O}$. The error in the measurements of the mean velocity is estimated to be less than $1 \%$, and the error of the mean temperature is expected to be less than $10 \%$. The error in the measurements in $\mathrm{CH}_{4}, \mathrm{CO}_{2}$ and $\mathrm{CO}$, is between $8 \%$ and $15 \%$, and the error regarding the $\mathrm{O}_{2}, \mathrm{OH}$ and $\mathrm{H}_{2} \mathrm{O}$ is within $20 \%$ to $25 \%$ [54]. Regarding the pilot temperature, strong heat losses to the burner surfaces were reported. To account for these heat losses a modified pilot temperature $T_{p}$ different from the adiabatic flame temperature $T_{a d}=2248 \mathrm{~K}$ is introduced to account for these heat losses and to match the experimental measurements at the first station. Calculations on the same configurations have been reported with pilot temperatures of $1936 \mathrm{~K}$ [55], $2005 \mathrm{~K}$ [26] and $1785 \mathrm{~K}[24,26,30]$, which corresponds to losses of up to $20 \%$. The temperature uncertainty in the pilot is important and is possibly the largest source of error due to the strong dependence of chemistry on temperature [26]. Another followed approach [11] was to modify the enthalpy in the inflow streams in order to match the experimental temperature at the first experimental station which is very close to the nozzle at $x / D=0.25$. In the present work, the pilot inflow temperature is taken to be $1785 \mathrm{~K}$. Nevertheless, the temperature is compared to experimental data in the form of a non-dimensional progress variable, which is defined by $c=\left(T-T_{\text {air }}\right) /\left(T_{a d}-T_{\text {air }}\right)$ [54]

Table 3. Estimate of Time and Length Scales of the Flames under study [54] at the centre of the nozzle exit.

\begin{tabular}{|ccccccccccc|}
\hline Flame & $\boldsymbol{U}_{\boldsymbol{0}}$ & $u^{\prime}$ & $\tau_{c}$ & $\tau_{t}$ & $\varepsilon$ & $\tau_{\eta}$ & $\delta$ & $\eta$ & $l_{q}$ & $l_{t}$ \\
\hline & $(\mathrm{m} / \mathrm{s})$ & $(\mathrm{m} / \mathrm{s})$ & $(\mathrm{ms})$ & $(\mathrm{ms})$ & $\left(\mathrm{m}^{2} / \mathrm{s}^{2}\right)$ & $(\mathrm{ms})$ & $(\mathrm{mm})$ & $(\mathrm{mm})$ & $(\mathrm{mm})$ & $(\mathrm{mm})$ \\
\hline $\mathrm{F} 1$ & 65 & 8.45 & 0.44 & 0.51 & $4.50 \mathrm{E}+04$ & 0.04 & 0.175 & 0.05 & 1.9 & 2.4 \\
\hline $\mathrm{F} 2$ & 50 & 6.5 & 0.44 & 0.65 & $2.00 \mathrm{E}+04$ & 0.06 & 0.175 & 0.06 & 1.3 & 2.4 \\
\hline $\mathrm{F} 3$ & 30 & 3.9 & 0.44 & 1.1 & $4.00 \mathrm{E}+03$ & 0.13 & 0.175 & 0.1 & 0.6 & 2.4 \\
\hline
\end{tabular}

Table 3 shows an estimate of the time and length scales for the investigated flames at the centre of the nozzle exit [54]. This helps to locate their behaviour in the premixed combustion diagram. The chemical (or flame) time scale $\tau_{c}=\delta / s_{L}^{0}$ is based on the laminar burning velocity $s_{L}^{0}=0.4 \mathrm{~m} / \mathrm{s}$ and the laminar flame thickness $\delta$. The Kolmogorov time scale, $\tau_{\eta}=\sqrt{v / \varepsilon}$, is calculated using a burning kinematic viscosity $v=7 \cdot 10^{-5} \mathrm{~m}^{2} / \mathrm{s}$. Finally, the Kolmogorov length scale was estimated using the classic definition $\eta=\left(v^{3} / \varepsilon\right)^{1 / 4}$ and the quench scale (which represents the size of an eddy that will quench the reaction zone and mix the scalar field) by $l_{q}=\left(\varepsilon \tau_{C}^{3}\right)^{1 / 2}$ (see [54] for details) 
Based on the above order of magnitude estimates, all flames are clearly in the thin reaction zone regime since $\tau_{t} / \tau_{c}>1$ and $\tau_{c} / \tau_{\eta}>1$ with Damköhler numbers between 1.2 (F1) and 2.5 (F3). The F1 flame is at the borderline of the distributed reaction zones and the F3 flame is closer to the flamelet regime. It should be noted that all these estimates can only be approximate and that values of $l_{t}, u^{\prime}$ and $\varepsilon$ change considerably within the flame as turbulence levels change considerably a lot across and along the jet. In Figure 2 the graph on the left shows the location of the three flames under study (based on the values given in Table 2) in the modified premixed turbulent combustion diagram regime [61]. The position is approximate and other researchers [58], reported them closer to the corrugated regime. In their work, Pitsch and Lageneste [11] proposed a new regime diagram for LES studies of premixed combustion (see Figure 2 right). They replace the integral length scale in the y-axis by the filter width (which is a characteristic sub-grid length scale). Therefore, the location in the new regime diagram depends on the filter size. For the x-axis, they used the Karlovitz number, which is the only quantity that is independent of the filter width, defined as

$$
K a=\left(\frac{l_{t}}{\delta}\right)^{-1 / 2}\left(\frac{u^{\prime}}{s_{L}^{0}}\right)^{3 / 2}=\left(\frac{\delta}{\eta}\right)^{2}
$$

Based on this definition, the Karlovitz number varies between 3.4-11 [56].

The solution domain (cylindrical grid) extends 15 jet diameters in the downstream direction and 5 diameters in the radial direction (or 10 diameters in total). This means that the grid length was $0.18 \mathrm{~m}$ and the grid radius was $0.06 \mathrm{~m}$. Two LES grids were used named hereafter named as 'fine' and 'coarse'. In the 'fine' grid, $164 \times 74 \times 42$ cells were used in the longitudinal, radial and azimuthal direction respectively, while in the 'coarse' mesh, $112 \times 56 \times 36$ grid points were used. In the fine grid, a cold simulation of the F2 and F3 flames was run, concentrating on the velocity and turbulent kinetic energy values to ensure that grid is enough for simulations. Grid stretching in the radial directions was applied to resolve the strong gradients that arise near the inlet and in the shear layer of the flame. The minimum mesh size in radial direction occurs at the shear layer and flame position where $\Delta \approx 0.71 \mathrm{~mm}$ for the coarse grid and $\Delta \approx 0.46 \mathrm{~mm}$ for the fine grid. For the fine grid, comparing the mesh size with the length scales given in Table 3, we observe that $\Delta / \delta \approx 2.62$ and $\Delta / \eta \approx 4.6-9.2$ (for the F3 and F1 flames respectively). For the coarse grid, comparing the mesh size with the length scales given in Table 3, it is derived that $\Delta / \delta \approx 4.05$ and $\Delta / \eta \approx 7.1-14.2$. In all the simulations in the present work, $\delta<\Delta<<l_{q}<<l_{t}$ for all three flames suggesting the fine grid resolution is enough to capture all the relevant thermo-physical phenomena. Since the mesh size is smaller than the quench length, the model will be able to capture the eddy that will quench the reaction zone and 'break' the flame. In the Figure 2, the corresponding regimes to the grids are shown in the Pitsch and Lageneste diagram [11]. 

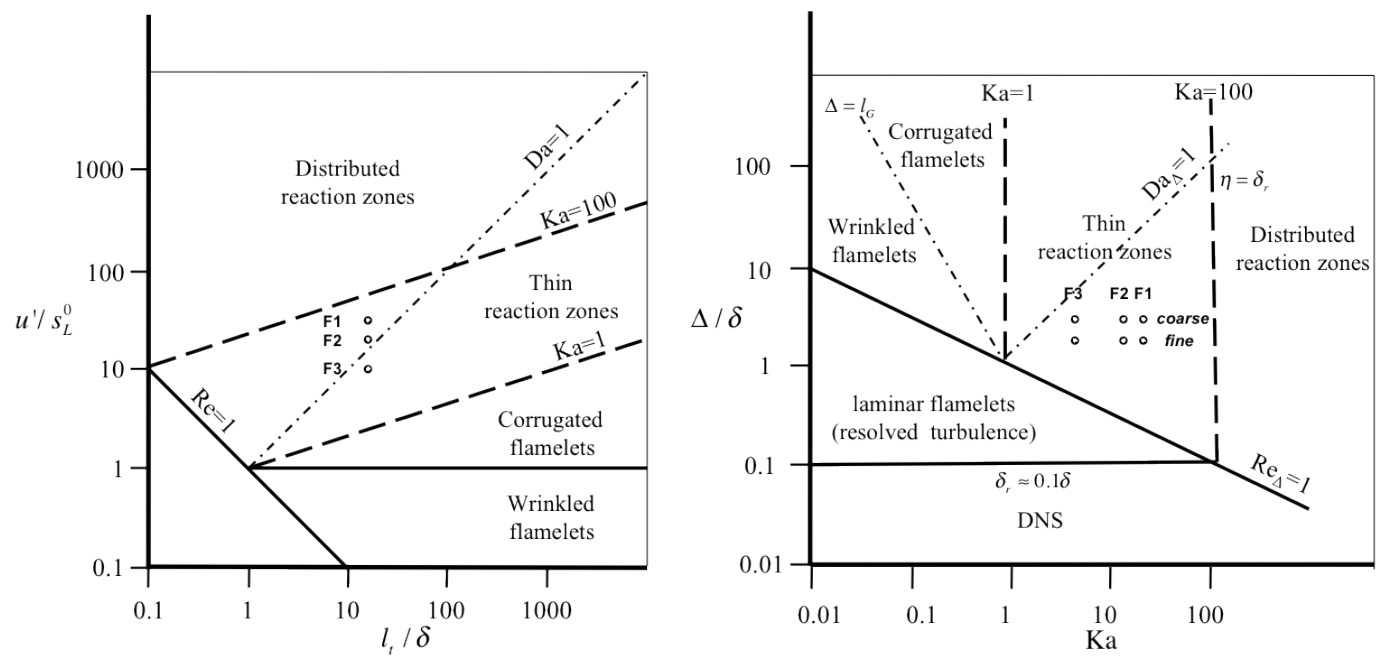

Fig. 2 The location of the three premixed flames under study in the classical combustion diagram (graph on the left) and in the new combustion diagram (graph on the right, in which the black dots denote the 'fine' grid locations and the red dots denote the 'coarse' grid locations).

Regarding the boundaries, free stream conditions have been employed for all lateral boundaries and a convective outflow condition has been applied at the outflow plane $[62,63]$. Azimuthal perturbations were superimposed to the mean inflow profiles to mimic inflow turbulence [64]. The associated mean velocity fluctuations were taken from turbulent kinetic energy measurements at the inlet of the flow. The method has proven to be successful in reproducing turbulent characteristics of jet flames [62,65]. To compute the chemical source term, a 19-species $\left(\mathrm{H}_{2}, \mathrm{H}, \mathrm{O}_{2}, \mathrm{OH}, \mathrm{H}_{2} \mathrm{O}, \mathrm{HO}_{2}, \mathrm{H}_{2} \mathrm{O}_{2}, \mathrm{CH}_{3}, \mathrm{CH}_{4}, \mathrm{CO}\right.$, $\mathrm{CO}_{2}, \mathrm{CH}_{2} \mathrm{O}, \mathrm{C}_{2} \mathrm{H}_{2}, \mathrm{C}_{2} \mathrm{H}_{4}, \mathrm{C}_{2} \mathrm{H}_{6}, \mathrm{NH}_{3}, \mathrm{NO}, \mathrm{HCN}$ and $\mathrm{N}_{2}$ ) 15-step reduced mechanism is used [66], derived from the complete GRI 3.0 skeletal mechanism using quasi-steady assumptions. The mechanism has been extensively validated and the laminar flame speed obtained with the chemical kinetics agrees very well with experimental data for the stoichiometric mixtures in this work. The 15-step mechanism solution will be compared against the 4-step global reaction scheme [67], which includes 7 species: $\mathrm{H}_{2}, \mathrm{O}_{2}, \mathrm{H}_{2} \mathrm{O}$, $\mathrm{CH}_{4}, \mathrm{CO}, \mathrm{CO}_{2}$ and $\mathrm{N}_{2}$. Both methods are able to reproduce accurately the laminar burning velocity at stoichiometric conditions. For reference, the original GRI 3.0 is also used for comparison purposes. All the transport properties, mixture viscosities and diffusivities are obtained from kinetic theory using Lennard-Jones potentials and standard mixing rules [68]. In the cases of equal diffusivities, a constant Schmidt and Prandtl number of 0.7 was assumed. If differential diffusivities are used, the thermal conductivity is obtained from kinetic theory and Blanc's mixing rule is used for the diffusivities.

The paper focuses on a parametric study of several simulation parameters. From modelling parameters: namely the grid resolution (coarse and fine), the number of fields (from $N=1$ to $N=16$ ) and the micro-mixing constants; together with the influence of the thermo-chemical conditions such as the pilot composition, the chemical kinetics and molecular transport. The base flame would be the F2 flame, although studies will be carried out for all three flames. 


\section{Results and Discussion}

\subsection{Flame Structure}

In order to validate the inflow conditions, a simulation with non-reactive conditions, with $T_{p}=T_{a i r}$ and $U_{p}=U_{a i r}$ was first performed without combustion modelling. The results are shown in Figure 3, where the resolved turbulent kinetic energy for the F2 and F3 cases is compared to experimental values. The figure shows an excellent prediction of both flames in the first three axial stations and a slight under-prediction at the fourth axial station. The axial velocity (not shown) shows good agreement at all stations. This under-prediction can be attributed to the fact that as the radial position increases further away from the main jet diameter, the cell size is slightly increased. Nevertheless, the above analysis verifies that the fine grid is able to capture the turbulent kinetic energy of the cold case accurately and that the discretization of the fine grid used for the simulations is enough for accurate turbulent predictions.
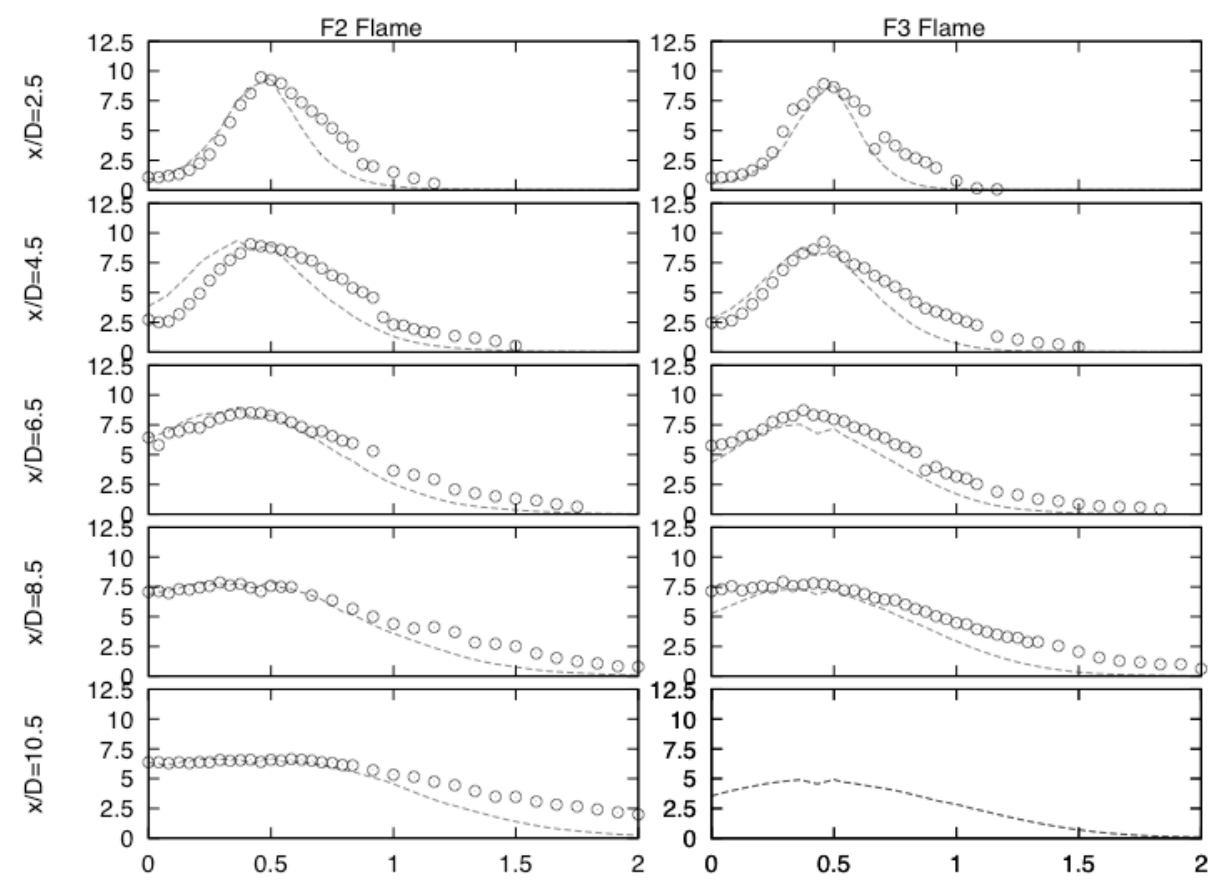

Fig. 3 Radial distribution of turbulent kinetic energy $k / k_{0}$, for the non-reactive cases of F2 and F3.

In this section, the simulation results of all the three flames are discussed. The influence of several parameters on the results will be examined in the next sections. Figure 4 shows instantaneous snapshots of temperature for the three flames. They are taken from the 16 field, 15-step chemistry mechanism in the 'fine' grid simulation with the standard values of the parameters studied in this work. 

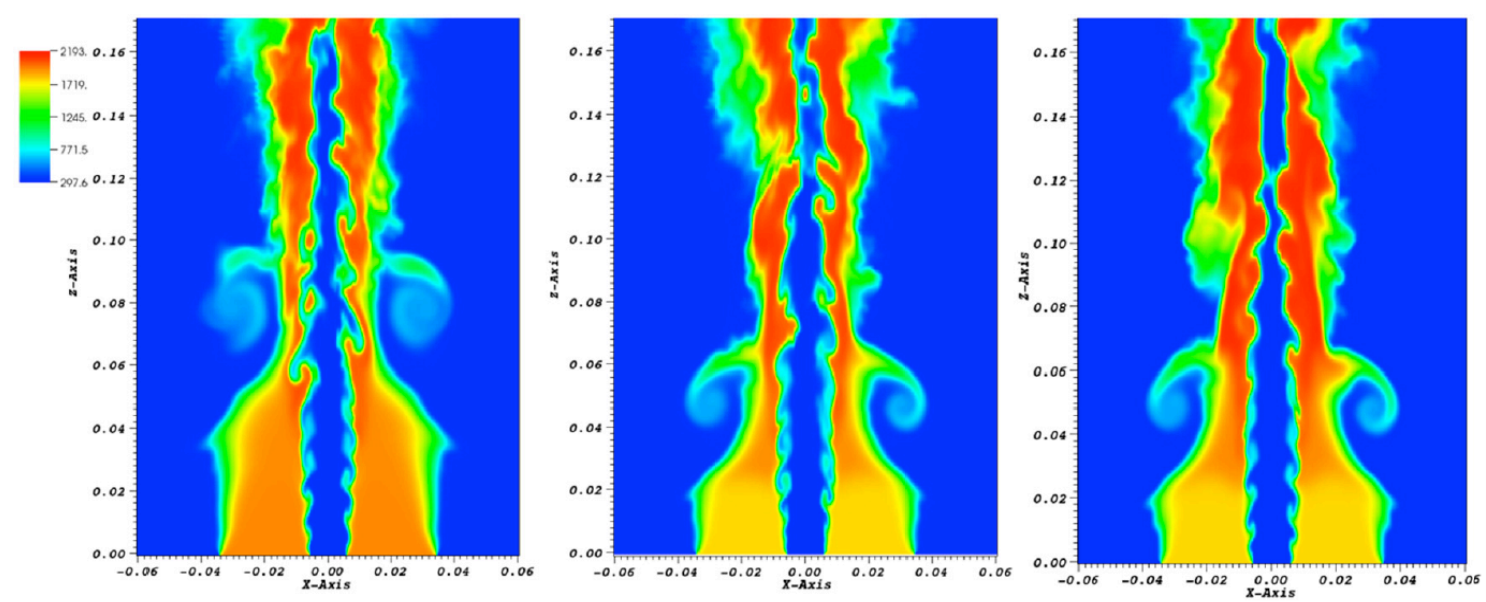

Fig. 4 Temperature plots of F1 (left), F2 (middle) and F3 (right) flames. The F1 flame exhibits thin and interrupted high temperature contours while the F3 flame exhibits an uninterrupted high temperature structure

The differences in the flame structure among the three flames are apparent. Contrary to the F3 flame (low Reynolds flame), the F1 flame exhibits thin and interrupted high temperature contours on the axial slice. The F3 flame on the other hand exhibits a thick and uninterrupted high temperature structure in the axial slice. The above observation suggests the proximity of the F1 flame to the distributed reaction zones regime (see Figure 2). In Figure 5, two temperature iso-surfaces for the F1 flame are shown. These surfaces are coloured with instantaneous $\mathrm{OH}$ values (left image) and mean $\mathrm{CO}_{2}$ values (right image). The 'holes' in the flame surface of the two images are apparent (blue regime on the $\mathrm{CO}_{2}$ plot and green regime in the $\mathrm{OH}$ plot). The interruption of the reaction is probably due to large scale mixing, indicating that the F1 flame is closer to the broken reaction regimes than the conventional distributed reaction regime (as observed for example in $[80,81]$ ) where intense burning is still present.
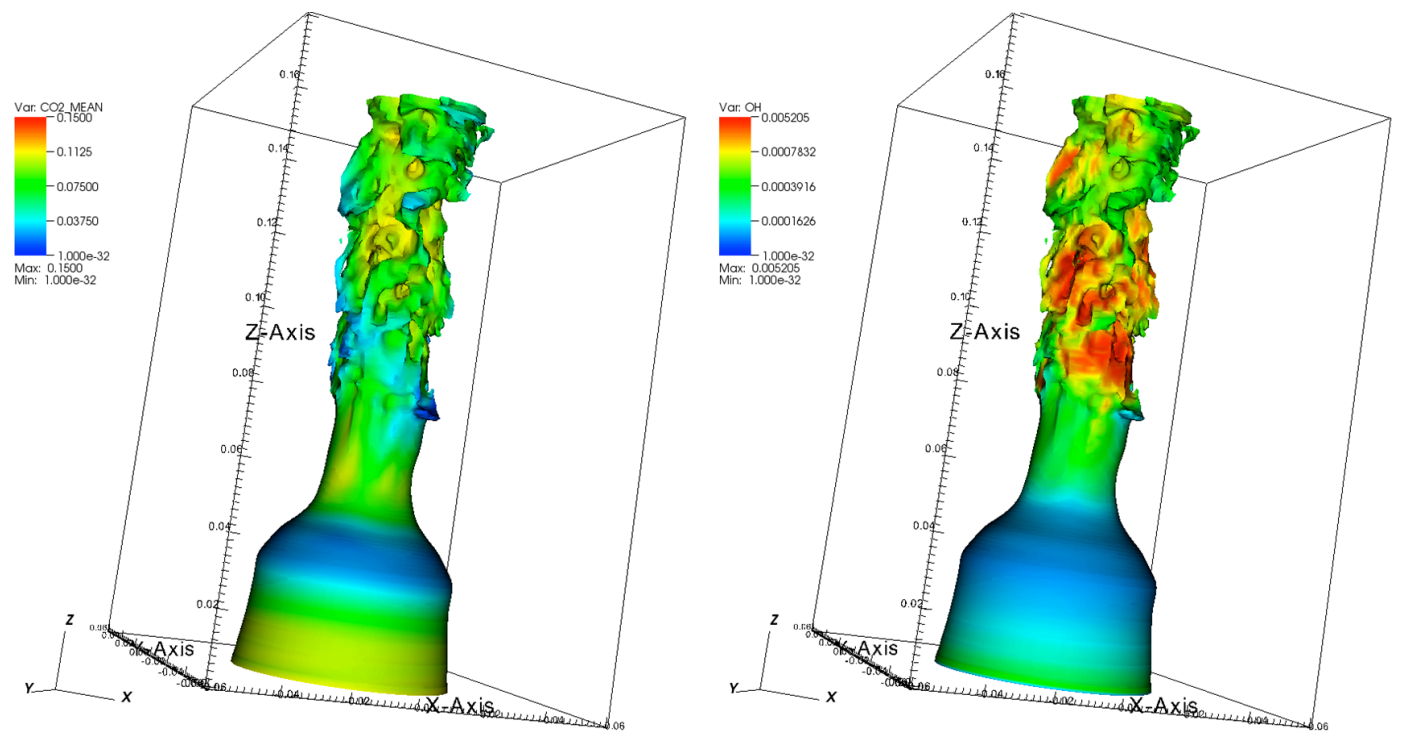

Fig. 5 Temperature iso-surface $(\mathrm{T}=1500 \mathrm{~K})$ with instantaneous $\mathrm{OH}$ (right) and $\mathrm{CO}_{2}$ values (left) coloured on the surface of the F1 flame. 
Figure 6 shows the curve-fitted mean flame-front position for the three flames. Based on experimental measurements [54], tabulated data of the temperature limits for the flame front at different axial positions are obtained. In all three flames, in the radial station (close to the nozzle exit), the flames extend radially to the edge of the outer nozzle $(6 \mathrm{~mm}$ in the radial direction). Further downstream, the flames burn inward, and as it would be expected, the nozzle exit velocity affects the radial position of the flame. At a given axial distance from the nozzle, the radial distance of the flame is larger in the F1 flame, which has the highest nozzle exit velocity. The figure shows a very good agreement between the LES-PDF simulated flame front and the experimental data, especially in the first radial stations of flames F1 and F2.
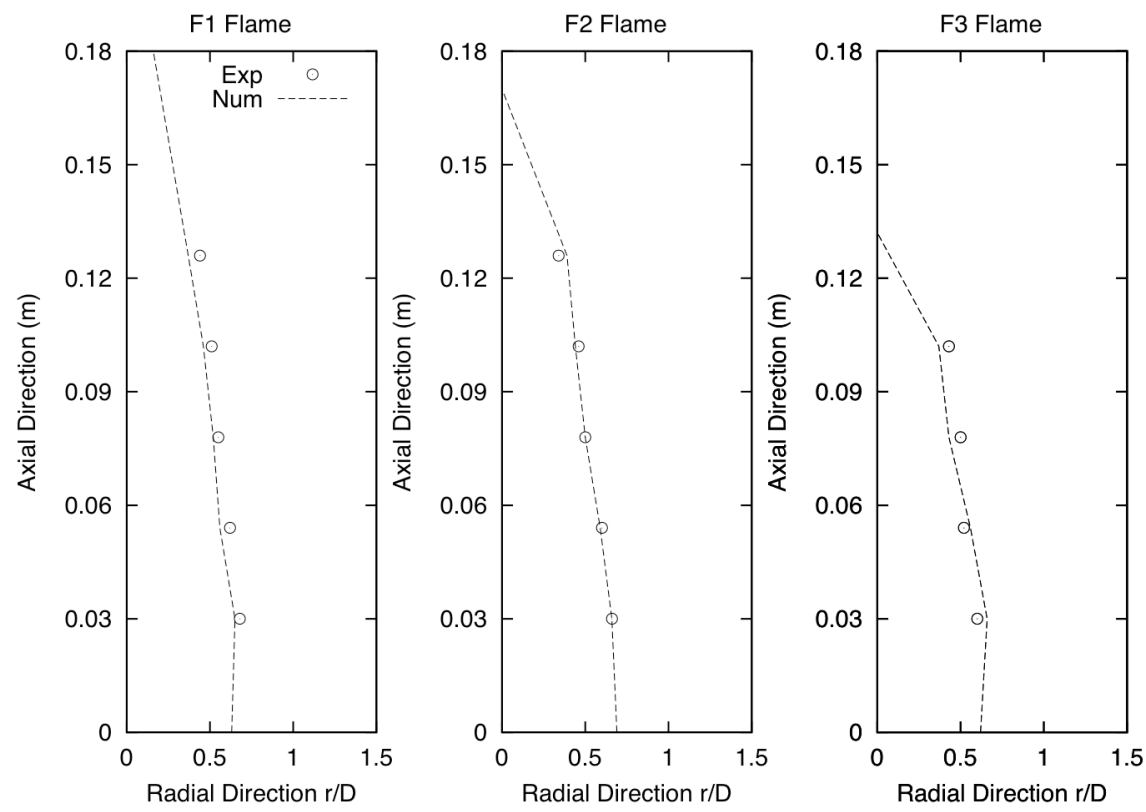

Fig. 6 Curve fitted mean flame front position of the three premixed flames. The dots denote experimental values (Chen et al.; 1996). The flame front is defined as the iso-line of the mean temperature of the temperature limits at each axial position [54]

The stochastic fields method allows to easily extract the instantaneous sub-grid PDF. In Figure 7, the $\mathrm{CO}_{2}$ mass fraction marginal PDFs are shown for the three flames at the flame front region at two axial locations. The histograms were obtained by 'binning' the instantaneous field values of a number of cells located very close to the flame front and at two axial positions (shear layer where the highest temperature gradients are observed). Despite the fact that this is only an instantaneous plot of a modelled PDF, it can give a qualitative indication of the flame regime. Regarding the first axial position, in the F1 flame the majority of the values is concentrated around 0.04 , indicating that the flame has large probability of burning away from equilibrium (equilibrium $\mathrm{CO}_{2}$ mass fraction is 0.15). The exact regime of the flame cannot be directly extracted from the PDF, as all sub-grid scales are modelled. However such distribution suggests that locally and instantaneously, the flame belongs to the distributed or broken reaction zones regime. The F2 flame shows approximately the same image in the first axial position, suggesting that even though the flame globally is not close to the distributed or broken reaction zone regime it can be so locally. Finally, the F3 flame in the first axial position shows a more uniform distribution. Regarding the second axial position, it is observed that as we move 
from the F1 flame towards the F3 flame, the variance diminished and PDF becomes narrower, with the F2 and F3 flames in the burn side of the flame.

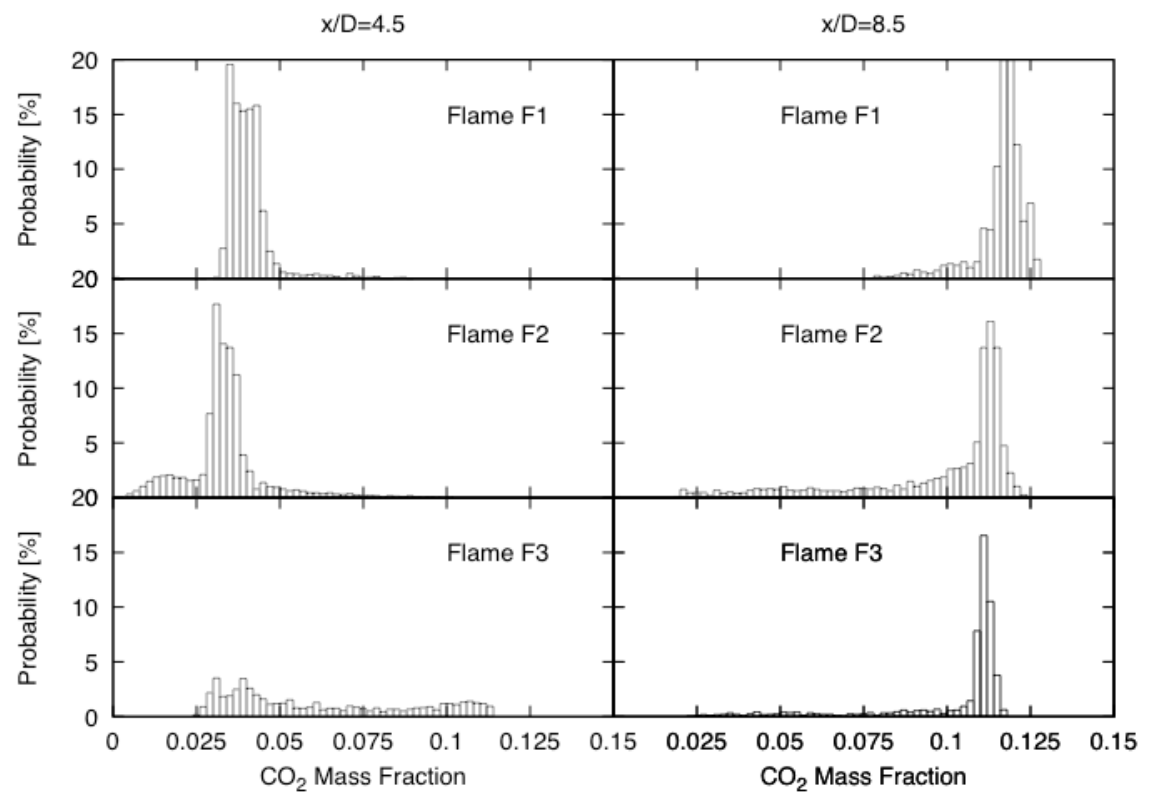

Fig. 7 Instantaneous sub-grid PDF $\mathrm{CO}_{2}$ mass fraction expressed as a probability distribution histogram in the mean flame front location at two axial positions for the F1-F3 flames

Figure 8 shows the scatter plot of the $\mathrm{OH}$ mass fractions as a function of the temperature, using a similar analysis than Duwig et al. [68]. The results show that the data follow an exponential distribution with very low values of $\mathrm{OH}$ mass fraction at low temperatures and a rapid increase around $1650 \mathrm{~K}$. As we move further downstream from the nozzle exit, the data points are highly scattered, indicating the effect of turbulence and entrainment of cold gases upon the reacting layer. Similar conclusions were presented in [68], where LES simulation results of a piloted lean premixed jet flame were compared to experimental measurements. At the same axial station, the scattering is broader in the F1 flame (with higher $\mathrm{OH}$ mass fraction values) with large deviations from the 1D laminar flame structure, suggesting the F1 flame is strongly affected by turbulence and close to a broken reactions zone.. 

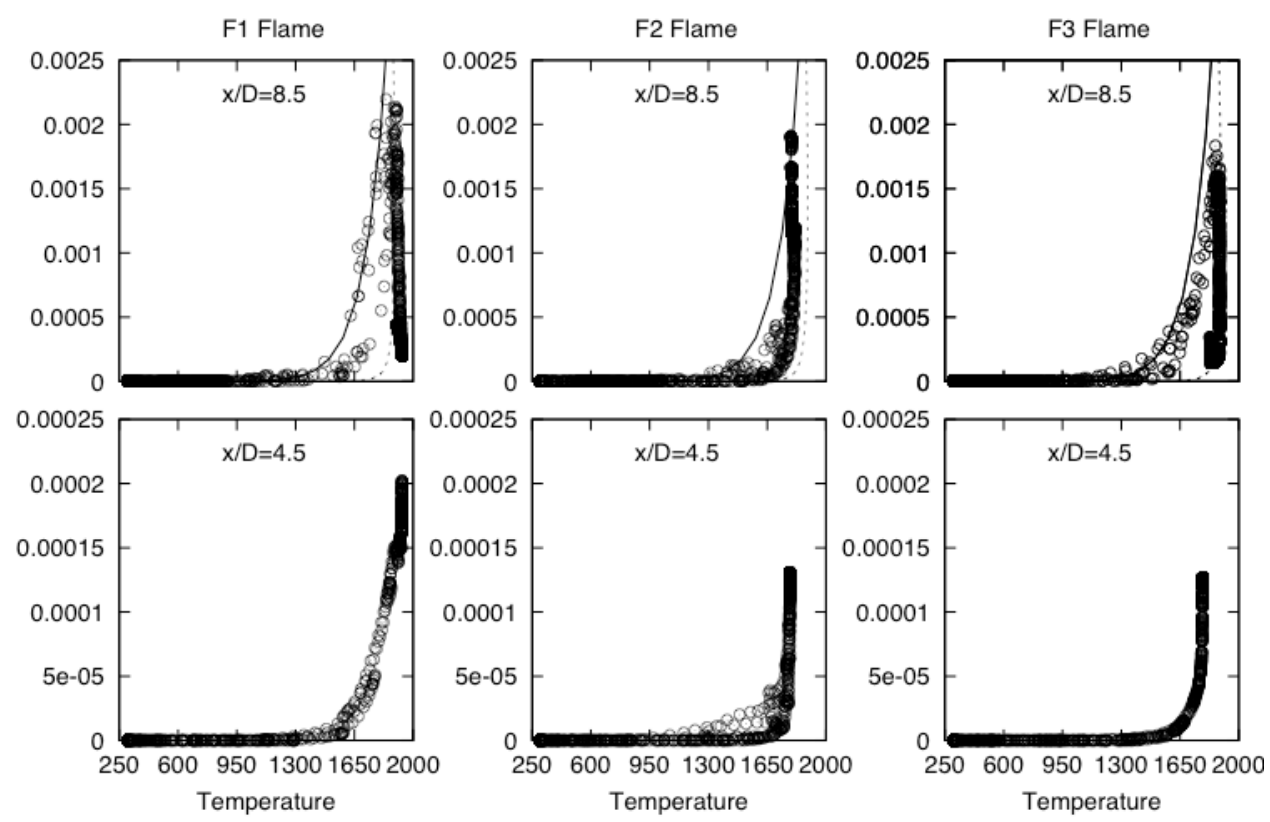

Fig. 8 Scatter plot of $\mathrm{OH}$ mass fraction as a function of temperature at three axial positions. The solid line is a 1D Stoichiometric premix flame, the dotted line is the rich branch of a diffusion flame at a strain rate of $40 \mathrm{~s}^{-1}$

\subsection{Sensitivity Analysis: Grid Resolution}

Figures 9 and 10 show the effects of the grid resolution on the velocity and progress variable with $N=1$ (no sub-grid model). Unsurprisingly, the axial velocity is captured accurately with the fine grid in the all flames and is an improvement over the coarse mesh. Exceptions are found in the further downstream position of the F2 flame. In general, the F3 flame seems to be much more sensitive than the other two flames in grid refinement, especially in the shear layer area. In general, coarse meshes underestimate the turbulent flame speed and since the velocities of the F3 flame are lower than the other two flames, the relative error attributed to grid refinement must be proportionally larger than the other two flames. The levels of the turbulent kinetic energy and the progress variable are much better predicted in the 'fine' grid, as would be expected. The progress variable has a different behaviour (see Fig 10) and the benefits of grid refinement without model can mostly be seen only in flame F1. Introducing a measure of the error by calculating the difference between the LES and experimental data at $r / D=0.5$ (where experimental/numerical discrepancies are largest) and averaging over the three stations. The fine grid reduces the error from 11 to $3.7 \%$ in the F1 flame, while it increases it in the F3 flame (from 4 to $24 \%$ )

The same conclusion can be drawn for most of the species mass fractions with the only exception of CO (shown in Fig. 11). In both cases (both grids) for all the three flames, the $\mathrm{CO}$ mass fraction seems to be over-predicted, which agrees with previous findings $[24,25]$ and is unlikely that further refinement will lead to improvement in predictions. 


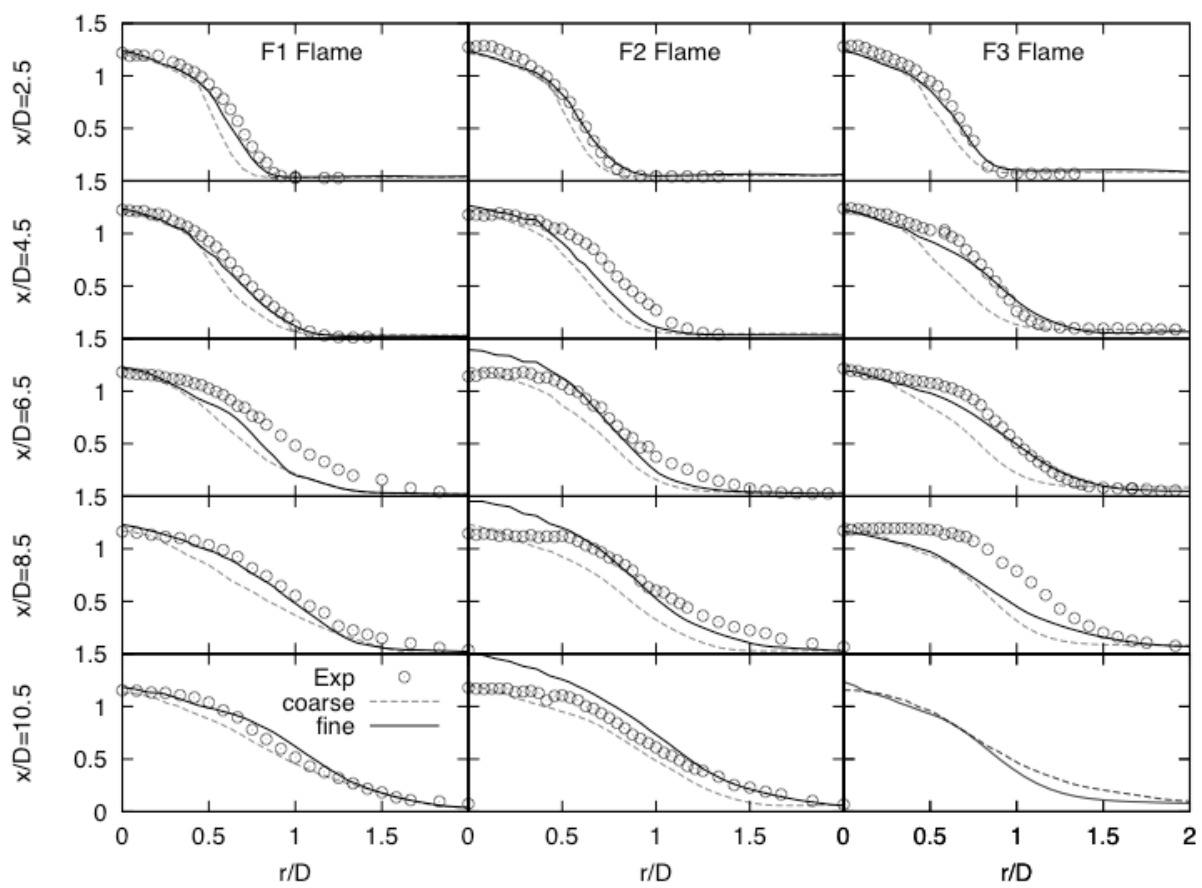

Fig. 9 Normalized axial velocity, $U / U_{0}$, radial distribution for the fine and coarse grids

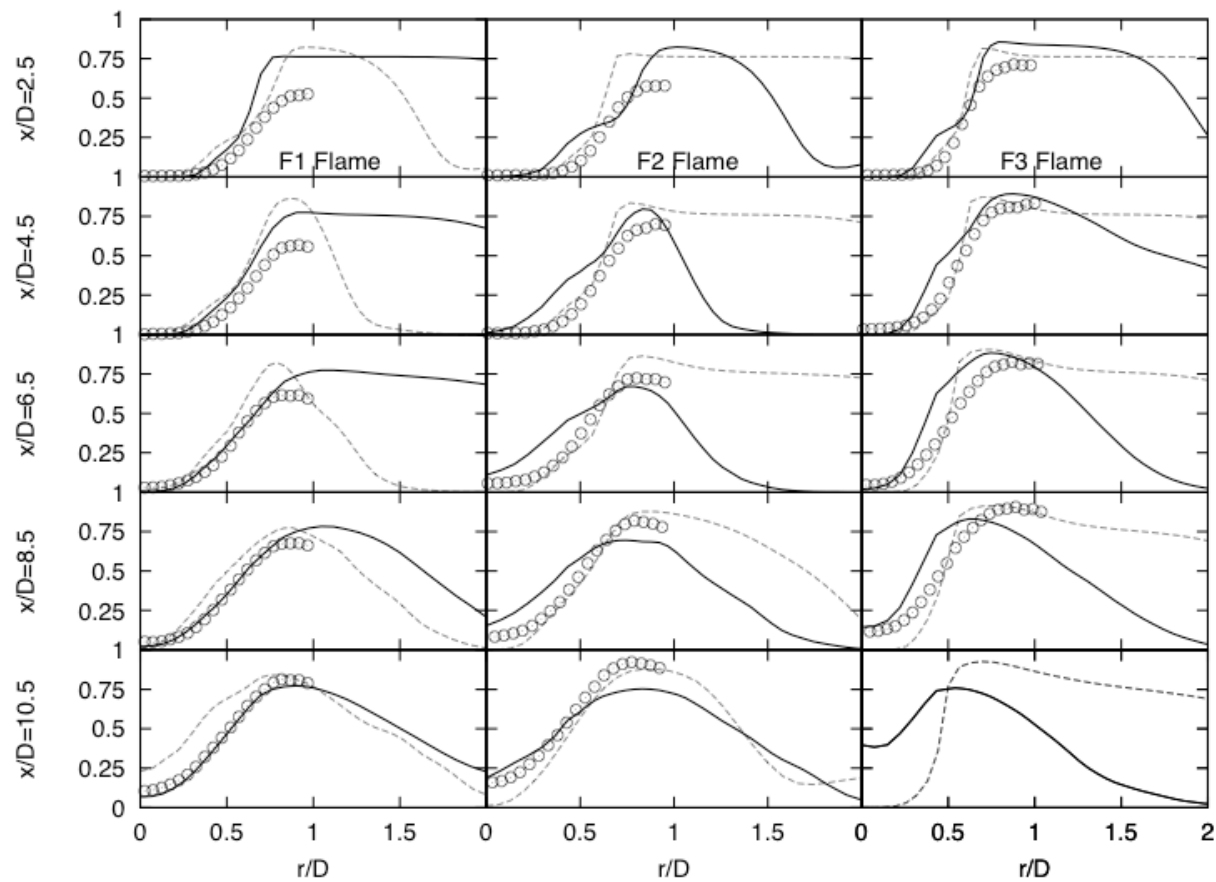

Fig. 10 Progress variable radial, $c$, distribution for the fine and coarse grids (Symbols as Fig 9) 


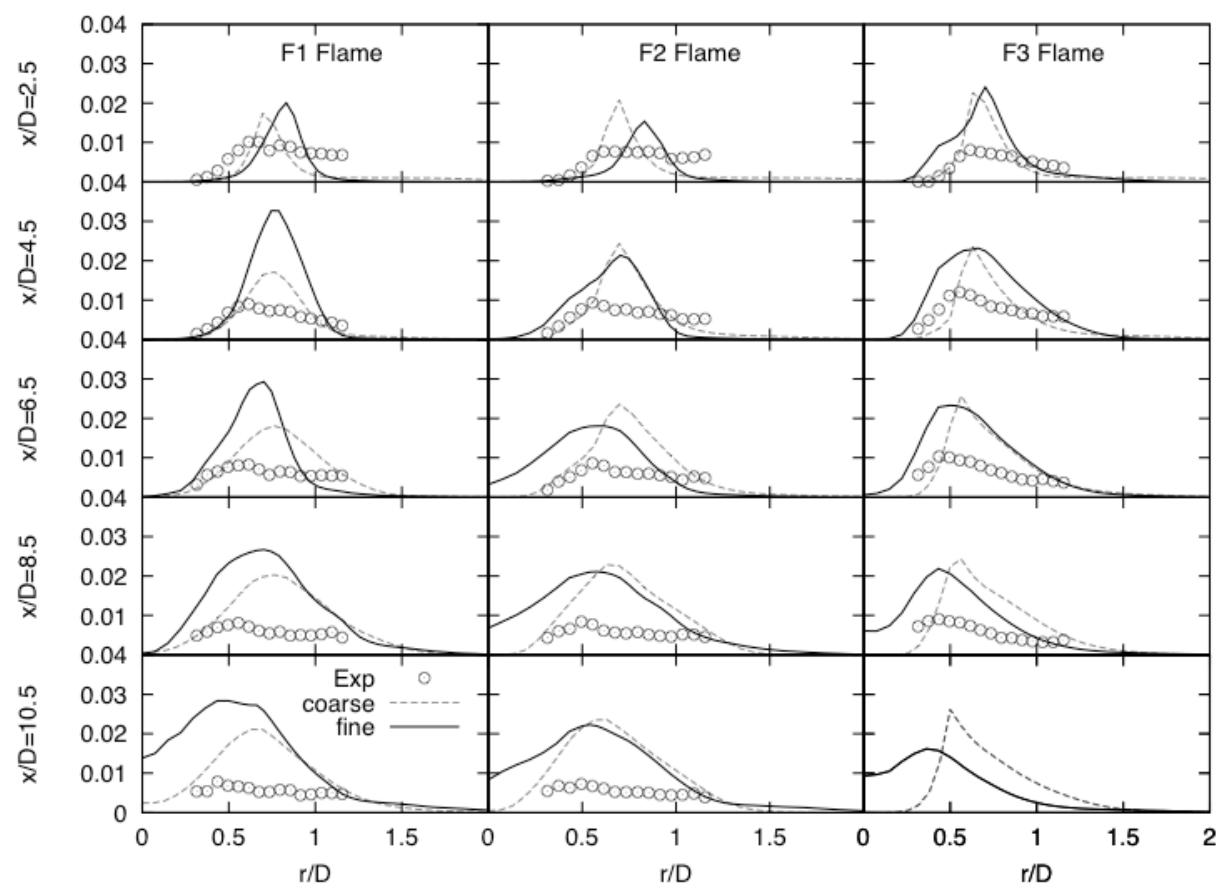

Fig. $11 \mathrm{CO}$ mass fraction radial distribution for the fine and coarse grids (Symbols as Fig 9)

\subsection{Sensitivity Analysis: Statistical Convergence}

In this section, the F1-F3 flames are investigated with 1, 4 and 16 fields. All these cases are run using the 'fine' grid and the 15 -step chemistry mechanism. It should be noted that the 1 field solution is a special case, as it indicates that the sub-grid PDF is a Dirac delta and in fact there is no sub-grid model. The combustion model itself reverts to a MILEStype approach [69-71]. Numerically, the accuracy of the temporal integration scheme also changes from $N=1$, to $N>1$. With $N=1$, the scheme revert to first order Euler scheme, while the actual solution of (14) with $N>1$ uses the Euler-Maramayama scheme [53] with accuracy $O(\sqrt{\Delta t})$. Nevertheless as the CFL number has been kept small (approximately 0.1 ), spatial accuracy is dominant. The stochastic error can be estimated as the variance of the predicted scalar divided by the square root of the number of fields. Therefore, in order to halve the sampling/statistical errors, the number of fields has to be quadrupled. 


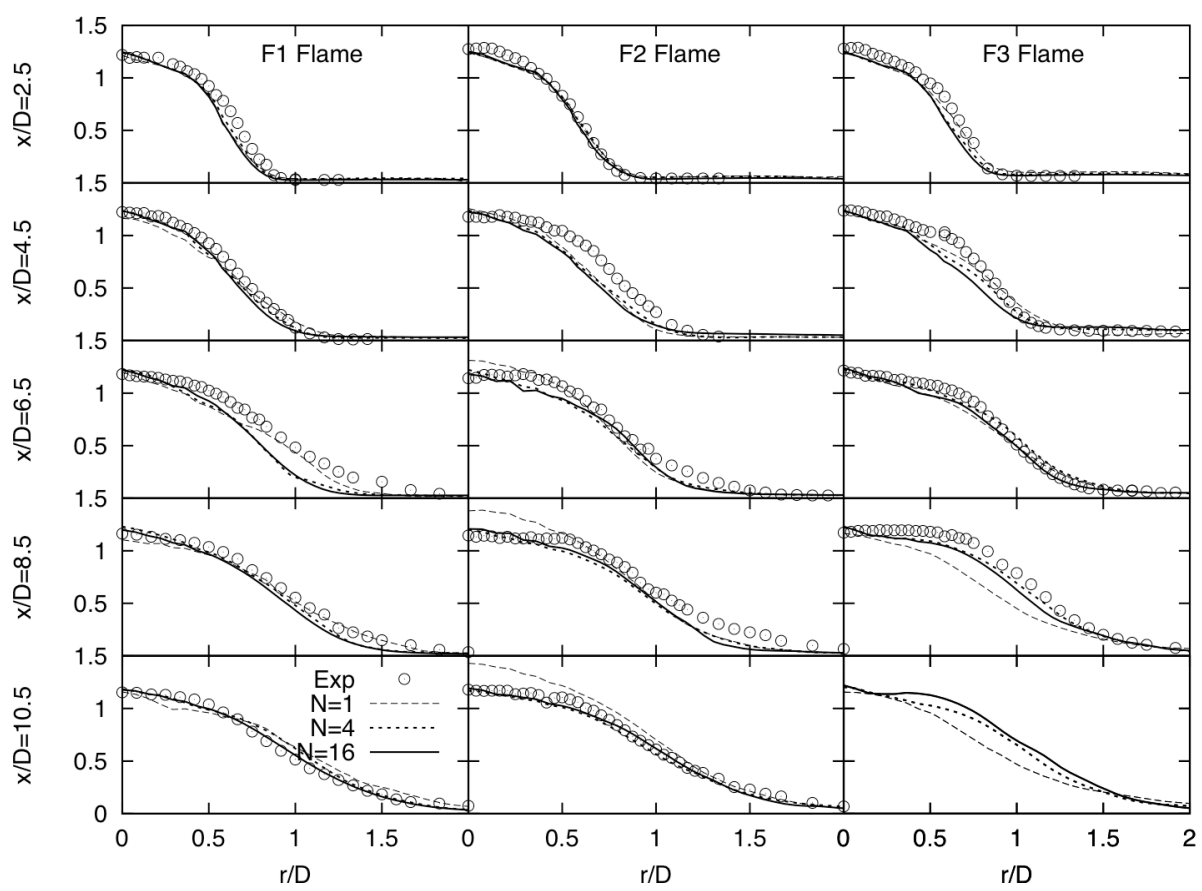

Fig. 12 Normalized axial velocity $U / U_{0}$, radial distribution with $N=1,4$ and 16

The effect of heat release on the flame structure is captured through the axial velocity distribution (see Fig 12). The increased number of fields improves the predictions in all three flames in a similar way as the grid refinement did in Fig 9. The biggest improvements can be seen in the F2 and F3 flames, in particular at $x / D>6.5$. It suggests that the sub-grid scale effects do indeed play some role in the calculations, especially in the low Reynolds F2 and F3 flames, despite the fact that in the high Reynolds number flame, the sub-grid scales are smaller (and stochastic fluctuations larger) and it would not be expected to be so accurately captured.

The sub-grid Damkhöler numbers [11] for the fine mesh can be estimated using the subgrid viscosity method [69] as 0.6 for flame F1, 0.8 (F2) and 1.35 (F3). In F3 the flame is not well resolved and there are large interactions between sub-grid scales and the flame and therefore sub-grid effects should be more accurately described. Flame F1 is better resolved $\left(D a_{\Delta}<1\right)$ and sub-grid modelling effects are limited [69]. 


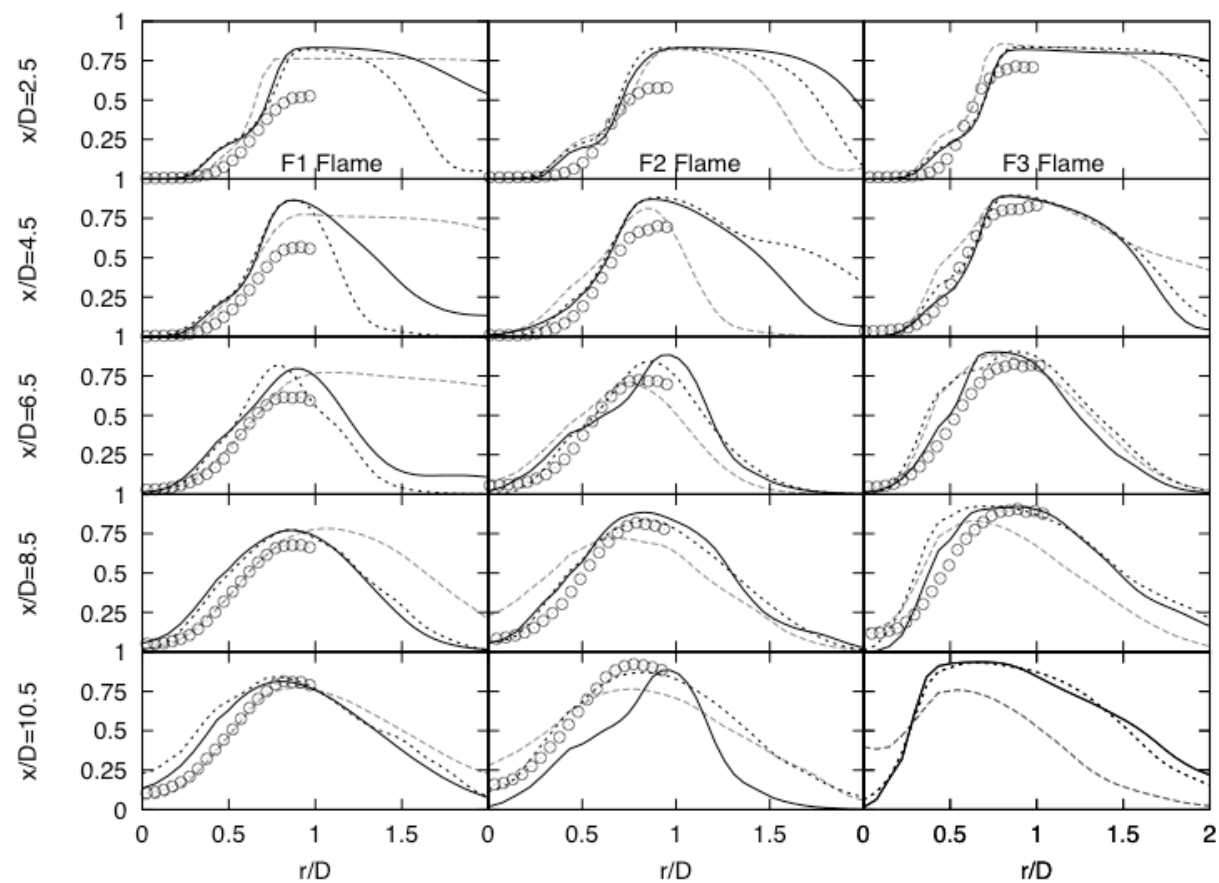

Fig. 13 Progress variable, $c$, radial distribution with $N=1,4$ and 16 . Symbols as Fig 12

The conclusions on Fig 13 are different. Overall there is relatively good agreement shown with $N=16$ (except at $x / D=10.5$ in flame F2). Using the same error measure as in the discussion of Fig. 10 (weighted error at $r / D=0.5$ ), the increase of fields affects the error differently for each flame. In the flame $\mathrm{F} 1$ the error in fact increases (from $12 \%$ with $N=4$ fields to $16 \%$ with $N=16)$ and underperforms the results with $N=1(3.7 \%)$. In flames F2 and F3, the sub-grid model have a large effect in the error, reducing it from $35 \%(N=1)$ to $7.4 \%(N=16)$ in the F2 flame and from $22 \%(N=1)$ to $7.8 \%(N=16)$. The increase in the number of fields reduces the error but less than $0.5 \%$ (from 7.9 to $7.4 \%$ ) in flame F2.

It seems that as the number of fields is increased, the flame is "thickened" and the flame speed prediction is improved in a similar way as the TFM model [9], similar interlinks between TFM and transport models where shown in [69]. In the TFM model, the modification of the reaction rate to capture the flame has to be compensated by adjustments in the diffusion terms. But as the number of fields is increased, this is achieved indirectly as the turbulent motion increases the diffusion of the flame. It has to be reminded that in stabilized flames such as the present burner, the flame movement is limited and the time averaging of the results may disguise instantaneous inaccuracies in the flame position. 

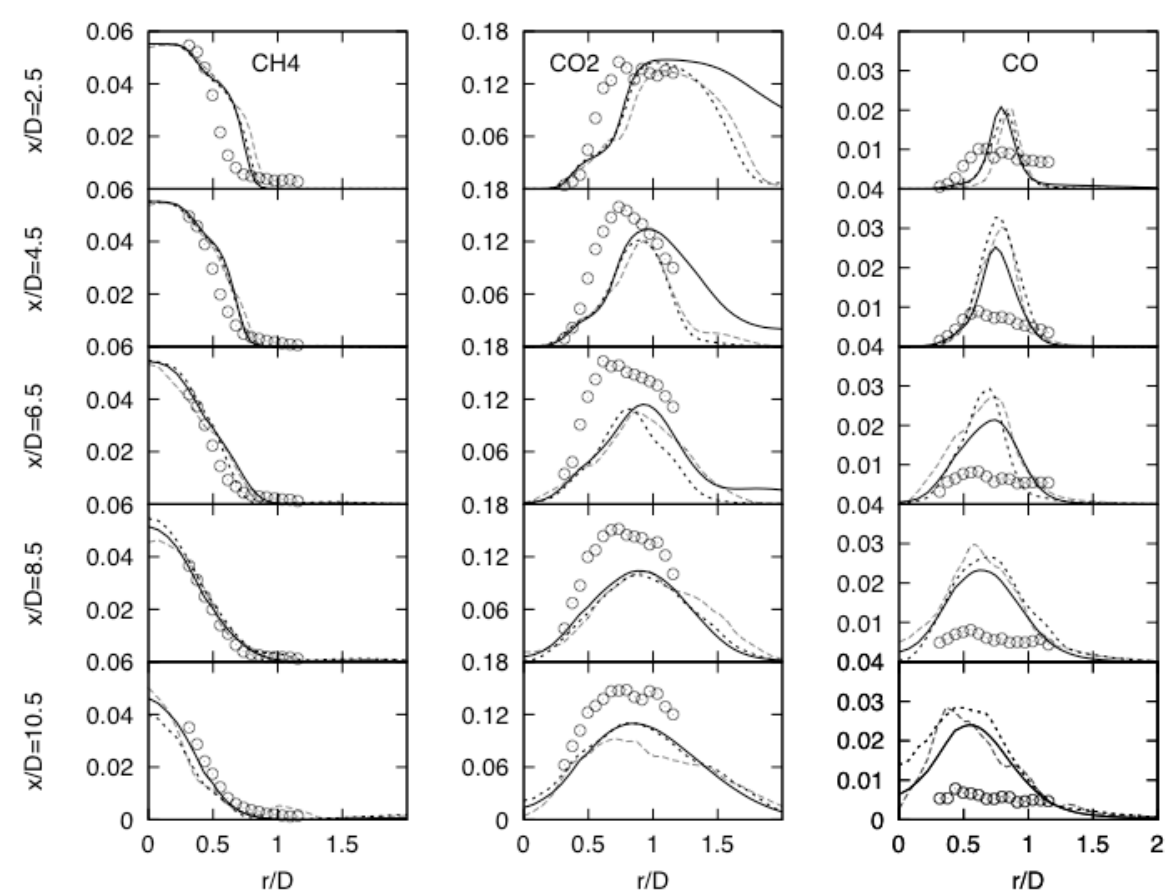

Fig. $14 \mathrm{CH}_{4}, \mathrm{CO}_{2}$ and $\mathrm{CO}$ radial distribution with $N=1,4$ and 16 . Flame F1. Symbols as Fig 12
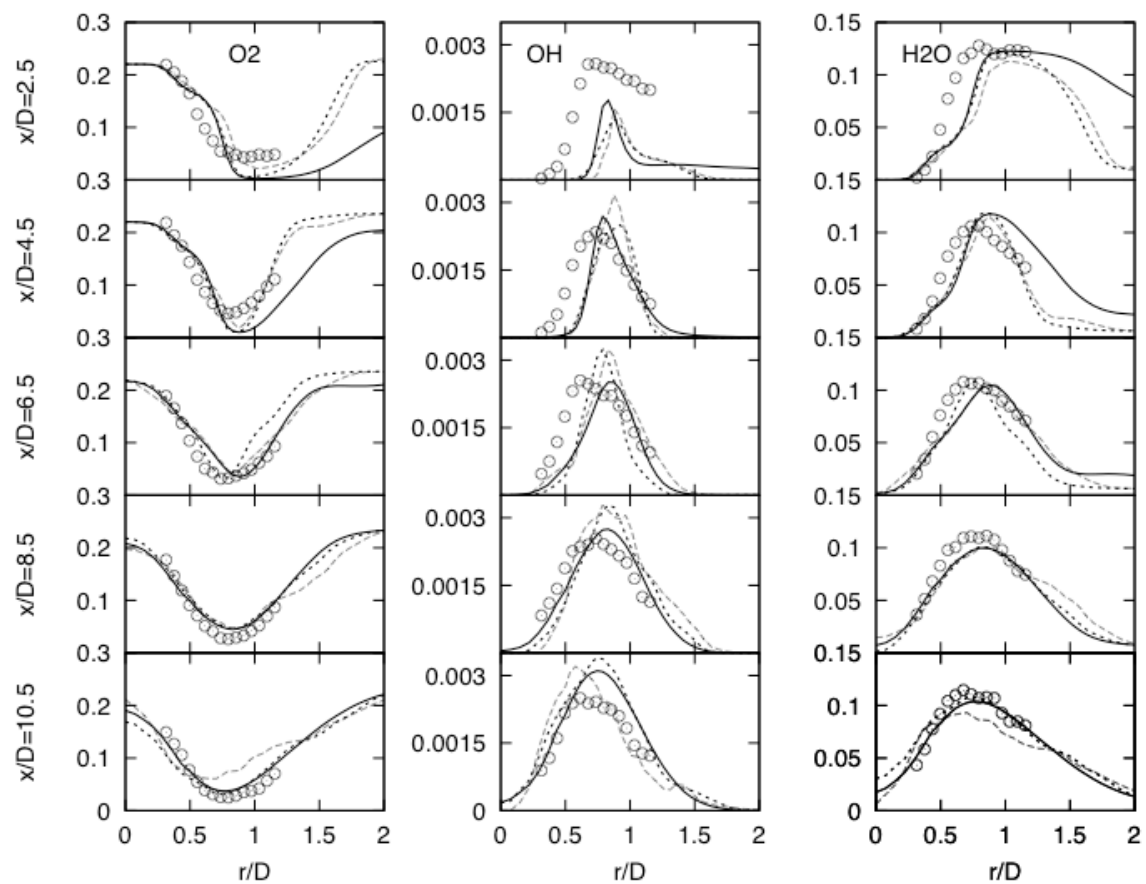

Fig. $15 \mathrm{O}_{2}, \mathrm{OH}$ and $\mathrm{H}_{2} \mathrm{O}$ radial distribution with $N=1,4$ and 16. Flame F1. Symbols as Fig 12 

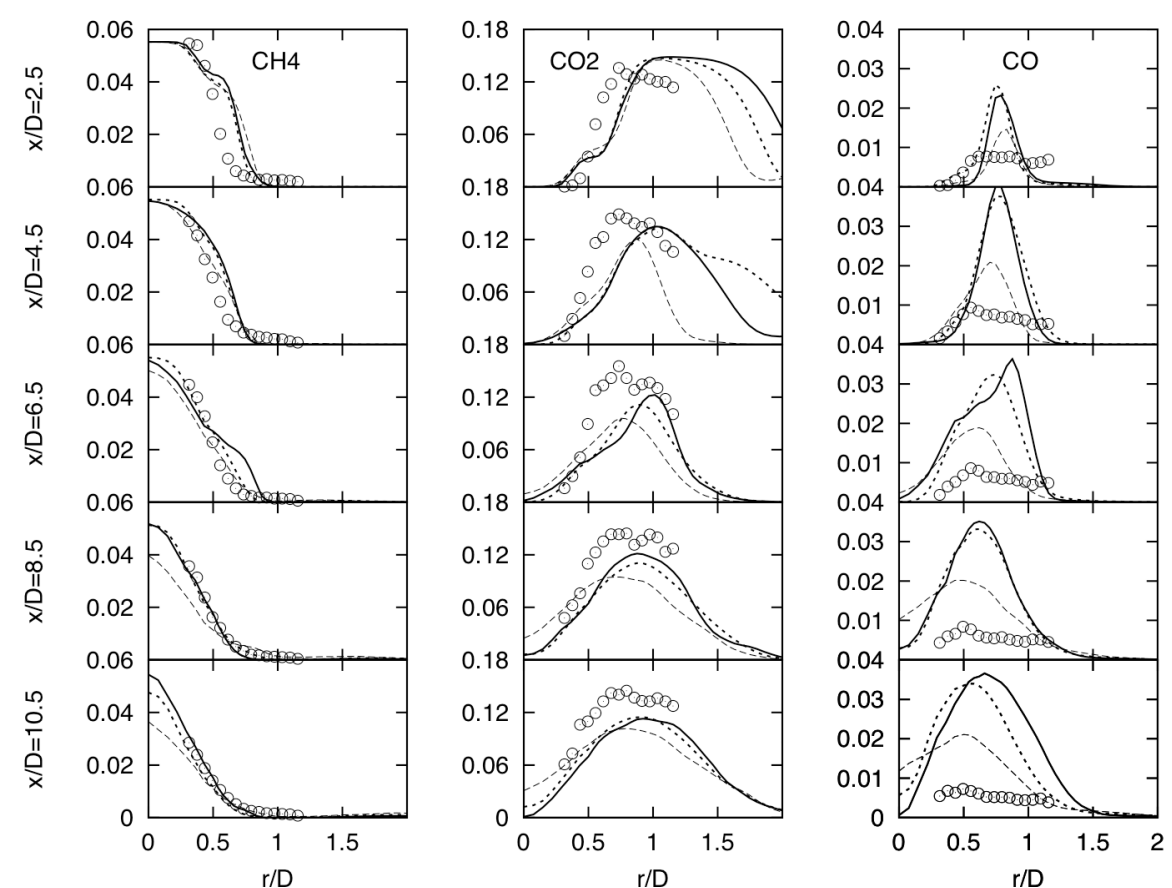

Fig. $16 \mathrm{CH}_{4}, \mathrm{CO}_{2}$ and $\mathrm{CO}$ radial distribution with $N=1,4$ and 16 . Flame F2. Symbols as Fig 12
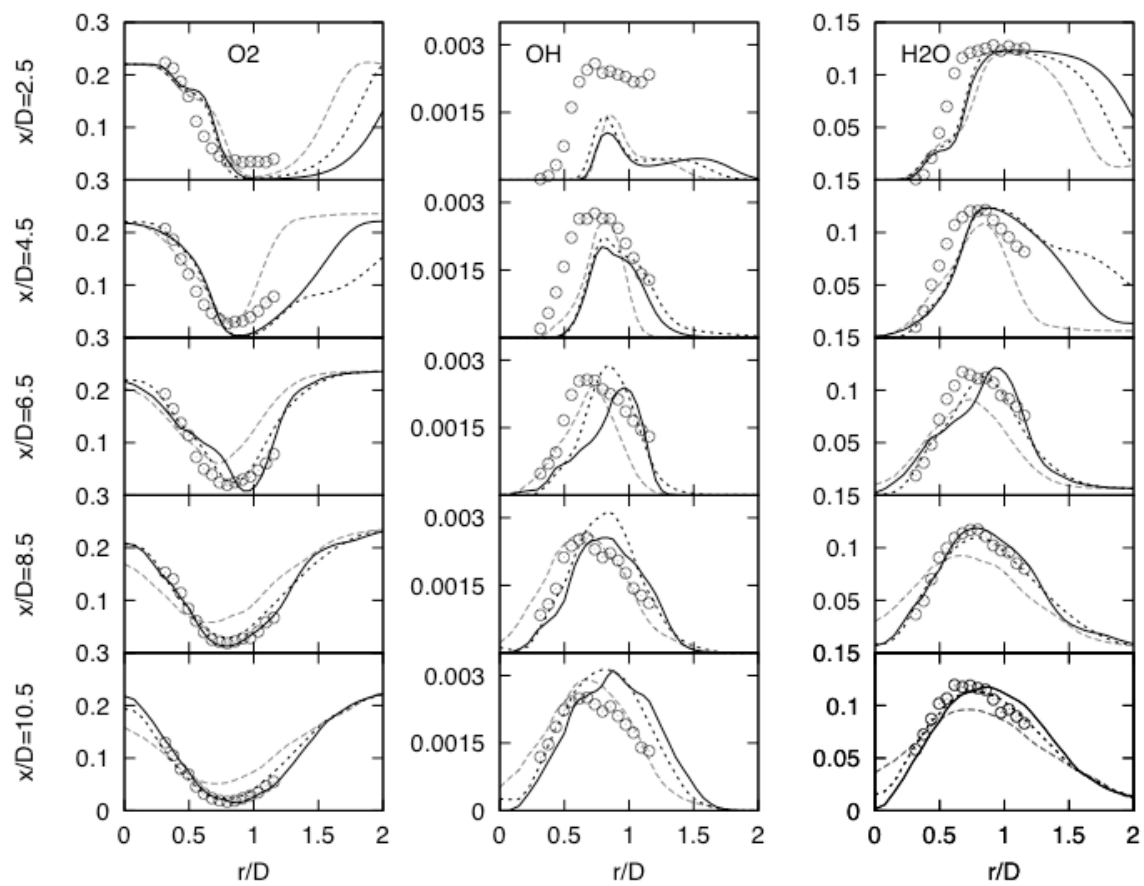

Fig. $17 \mathrm{O}_{2}, \mathrm{OH}$ and $\mathrm{H}_{2} \mathrm{O}$ radial distribution with $N=1,4$ and 16. Flame F2. Symbols as Fig 12 

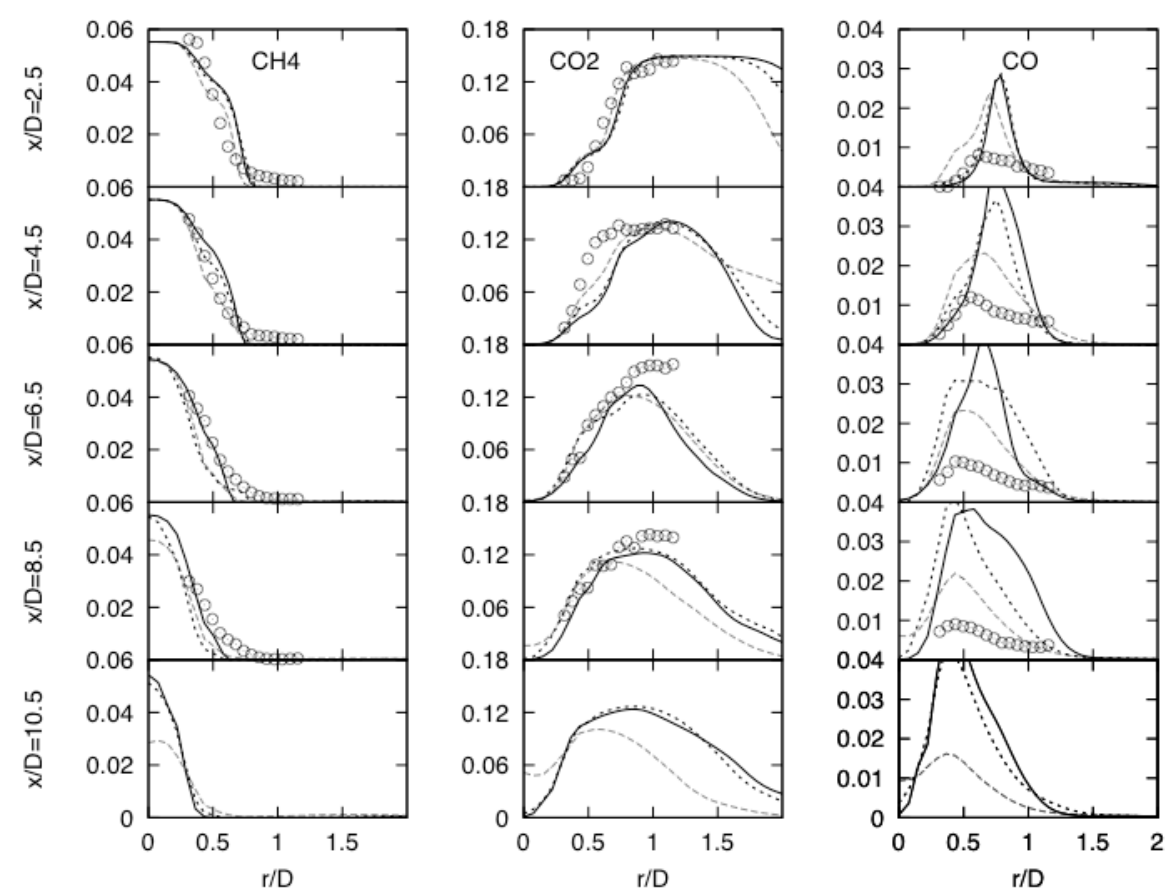

Fig. $18 \mathrm{CH}_{4}, \mathrm{CO}_{2}$ and $\mathrm{CO}$ radial distribution with $N=1,4$ and 16 . Flame F3. Symbols as Fig 12
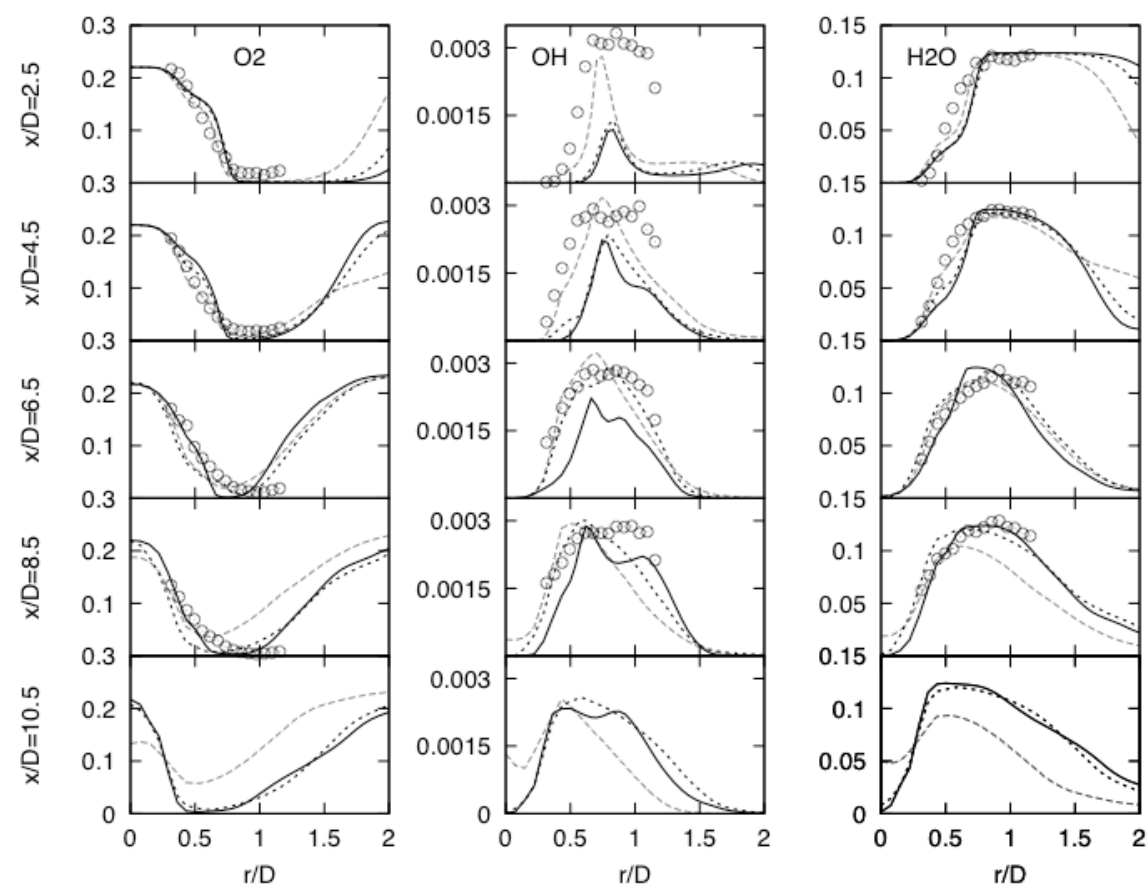

Fig. $19 \mathrm{O}_{2}, \mathrm{OH}$ and $\mathrm{H}_{2} \mathrm{O}$ radial distribution with $N=1,4$ and 16. Flame F3. Symbols as Fig 12

Species mass fractions have a different response to increase in the number of fields (see Figs. 14-19). The $\mathrm{O}, \mathrm{OH}$ and $\mathrm{H}_{2} \mathrm{O}$ predictions show slight improvement with $N$. The agreement between simulations and experiments is relatively good downstream, except underestimation of the $\mathrm{OH}$ at locations closer to the inflow. $\mathrm{CH}_{4}$ is generally predicted accurately in all three flames, the number of fields does not significantly alter the results except maybe at the F3 (Fig 18) at $x / D>6.5$ is predicted more accurately for the F3 flame (in accordance with RANS method) while for the F1 and F2 flames, a over-prediction can be observed close to the nozzle exit. $\mathrm{CO}_{2}$ present a more erratic behaviour with good results in flame F3 but under-prediction at F1 and F2. CO is over-predicted (Figs, 14, 16 
and 18) in all three flames and is difficult to establish a trend with increased $N$. It has been suggested [24] that the over-prediction of $\mathrm{CO}$ can explain the under-prediction of $\mathrm{CO}_{2}$ suggesting a slower oxidation rate in the $\mathrm{CO}$ to $\mathrm{CO}_{2}$ reaction. However different researchers $[24,25]$ reported the same discrepancies with experimental data (much larger than 10-25\% experimental uncertainties) using different chemical mechanisms. In order to examine the under-prediction of the $\mathrm{CO}$ mass fraction, a new progress variable was defined as follows:

$$
c^{*}=\frac{Y_{C O}+Y_{C O 2}}{\left(Y_{C O}+Y_{C O 2}\right)_{p i l o t}}
$$

The results are shown in Fig 20, the newly defined progress variable shows much better performance compared to experimental data (even in the stations close to the nozzle exit where a flaw in the pilot temperature can be observed). For example, in the station $x / D=8.5$, the simulation with $N=16$ was under-predicting $\mathrm{CO}$ and over-predicting $\mathrm{CO}_{2}$ (Fig 16). The results obtained with this new progress variable $c^{*}$ show better agreement than $c$ based on temperature (Fig 20 vs. Fig 13).

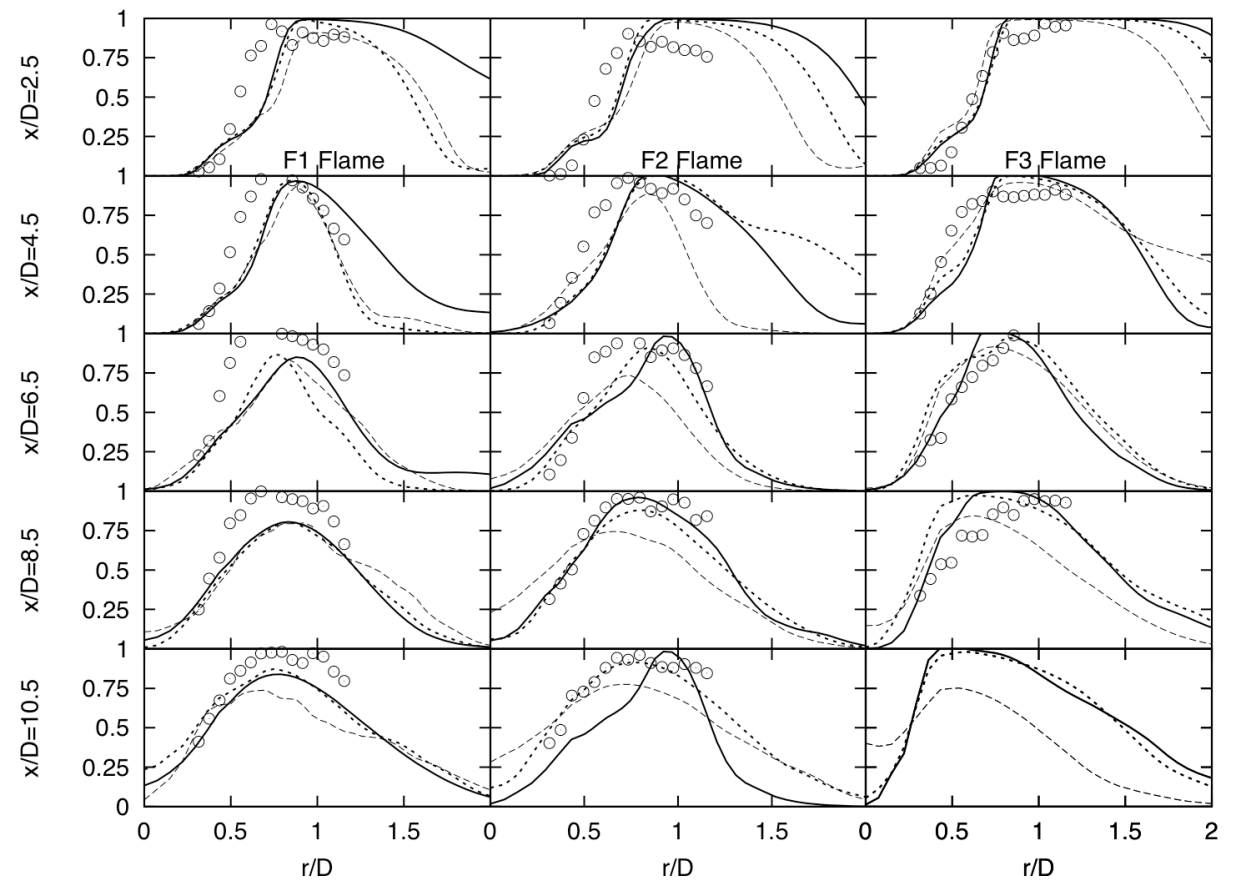

Fig. 20 New progress variable, $c^{*}$, radial distribution with $N=1,4$ and 16 . Symbols as Fig 12

\subsection{Sensitivity Analysis: Chemical Kinetics}

In this section, several methane combustion chemical mechanisms (see Section 3) were implemented in the F2 flame with $N=1$. The 15-Step and GRI3.0 mechanisms did not show major differences in the major species. Figure 22 shows the F2 results at the axial station with largest differences. The 15-step mechanism is a significant improvement over the 4-step mechanism. On the other hand, the differences between the 15 step and the GRI 3.0 mechanisms are much minor and this could justify the use of the much faster 15 step mechanism over the complete GRI3.0 mechanism. It should be noted that in the case of the F2 flame, with exactly the same settings (number of nodes, size of the domain, etc.), the 15 step mechanism required about 5 days to obtain converged statistics in a 4- 
core Intel Pentium Q6600 while the GRI 3.0 required about three weeks on the same machine. The 4-step mechanism, despite being the fastest, has large errors, in the prediction of the progress variable $c$, indicating that the flame speed is not accurately predicted. Even though the mechanism is able to capture correctly the laminar flame speed, it cannot predict the average flame position. CO errors with the 4-step mechanism have been reported in the literature [67] whereas the GRI3.0 mechanism gives slightly better $\mathrm{CO}$ and $\mathrm{H}_{2} \mathrm{O}$, but not large enough to explain the $\mathrm{CO}$ discrepancies . The larger differences occur, surprisingly at $r / D>0.7$ in the outer shear layer where the air/pilot shear layer interacts with the flame front (See Fig 3)
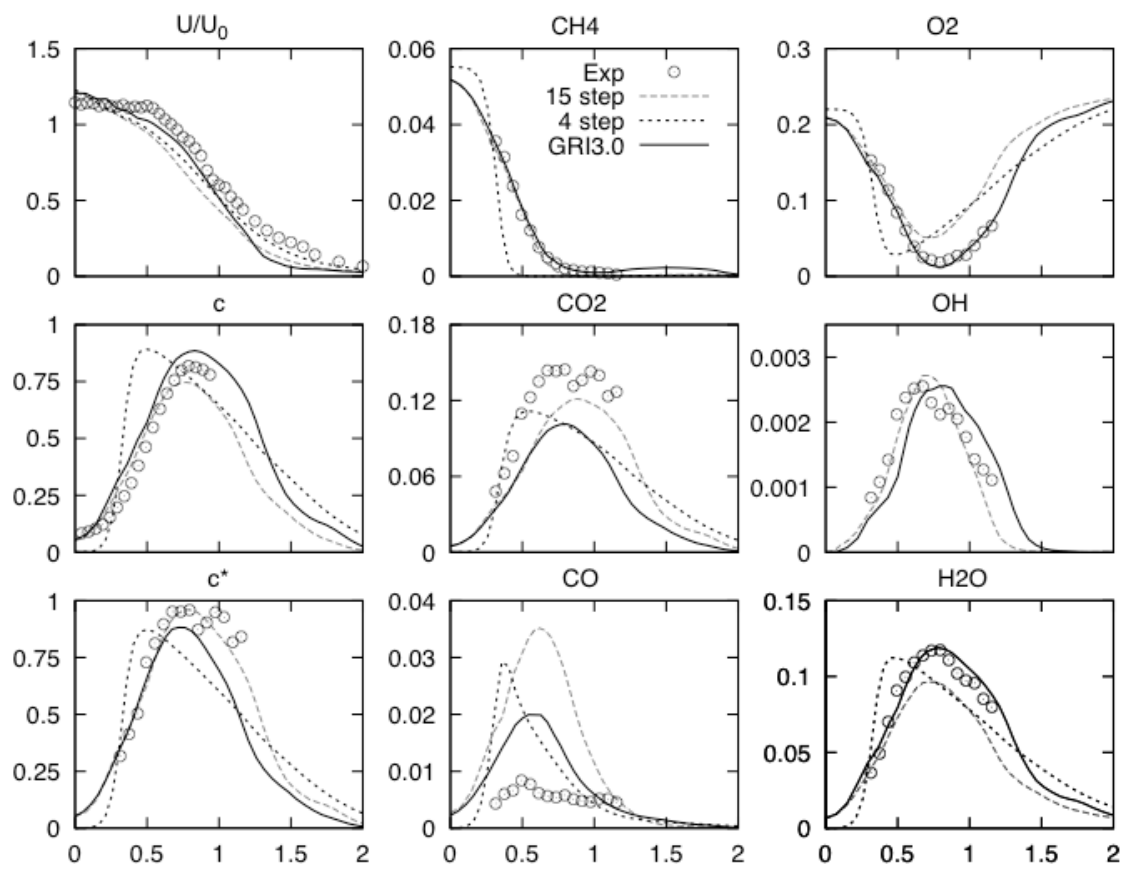

Fig. 21 Different chemical mechanisms predictions in the F2 flame at $x / D=8.5$

\subsection{Sensitivity Analysis: Micromixing}

As mentioned in the literature [41], the micromixing constant has a strong effect on RANS-PDF predictions, however it may not be so in LES due to considerably greater localization and reduced scalar variances. In this section the micro mixing effects are investigated in flame F2 with $N=4$. Four fields were selected as they presented the largest differences between them and therefore the model is more sensitive to the micromixing term. Three values $C_{\phi}=2,25$ and 80 were selected, without the scaling in Eq. (12). This range of values is much wider than similar studies done in RANS-PDF. The results show (see Fig 22) that the value of $C_{\Phi}$ does not significantly affect the flame structure. The temperature prediction is slightly improved with $C_{\Phi}=25$ and the same applies to the axial velocity results. $\mathrm{CO}$ results are largely unaffected. 

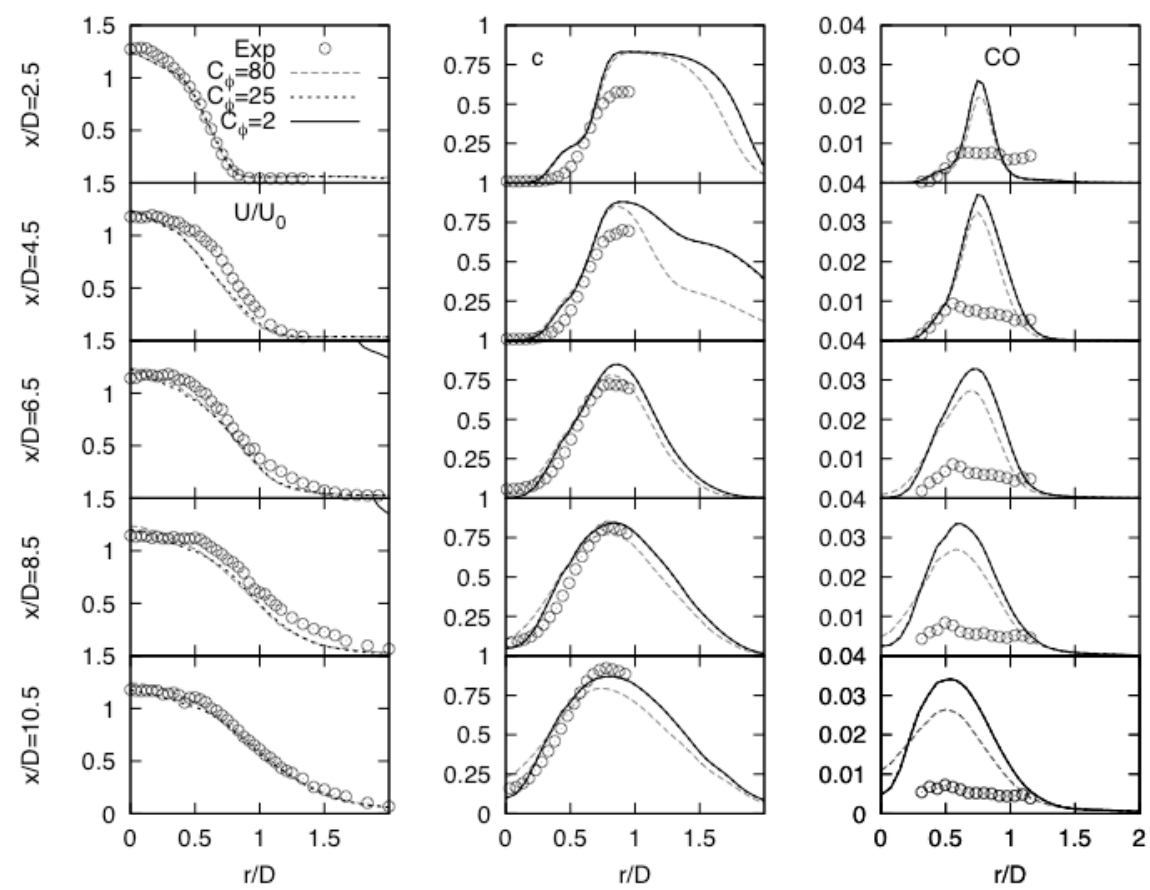

Fig. 22 Axial velocity $U / U_{0}$, progress variable $c$ and $\mathrm{CO}$ mass fraction distribution for the $\mathrm{F} 2$ flame with different $C_{\Phi}$

From RANS-type analysis $[42,72]$ it is expected that the $C_{\Phi}=2$ value would yield the most accurate results but large sensitivity is usually observed [73] . However, RANSPDF results on the same set of flames [57] revealed that the results depend significantly on its value. Similarly, Lindstedt and Vaos [25] applied the transported PDF model to the same flames using the coalescence dispersion model. In the F1 flame, by adopting a value of $C_{\Phi}>4$, a stable burning F1 flame was obtained. However, flame extinction occurred when $C_{\Phi}=2$ was selected (unlike the F2 and F3 flames). Fig 22 shows that, in LES-PDF it does not play a major role in the present conditions and its relative importance is expected to be less than in RANS simulations.

In Fig 23 a comparison is made between $N=1$ and the results obtained with $N=4$ and $C_{\Phi}=80$. The Figure shows that sub-grid effects do not disappear even at an unrealistic value of 80 and the mixing is improved (see axial velocity and $\mathrm{CH}_{4}$ distribution). The $\mathrm{CO}$ mass fraction prediction (which always shows the largest differences in these flames) still suggests that sub-grid scalar fluctuations play a part in CO production. Nevertheless, the above analysis (Figs 22 and 23) shows the LES-PDF approach is weakly dependent of $C_{\Phi}$ unlike RANS-PDF counterparts. 

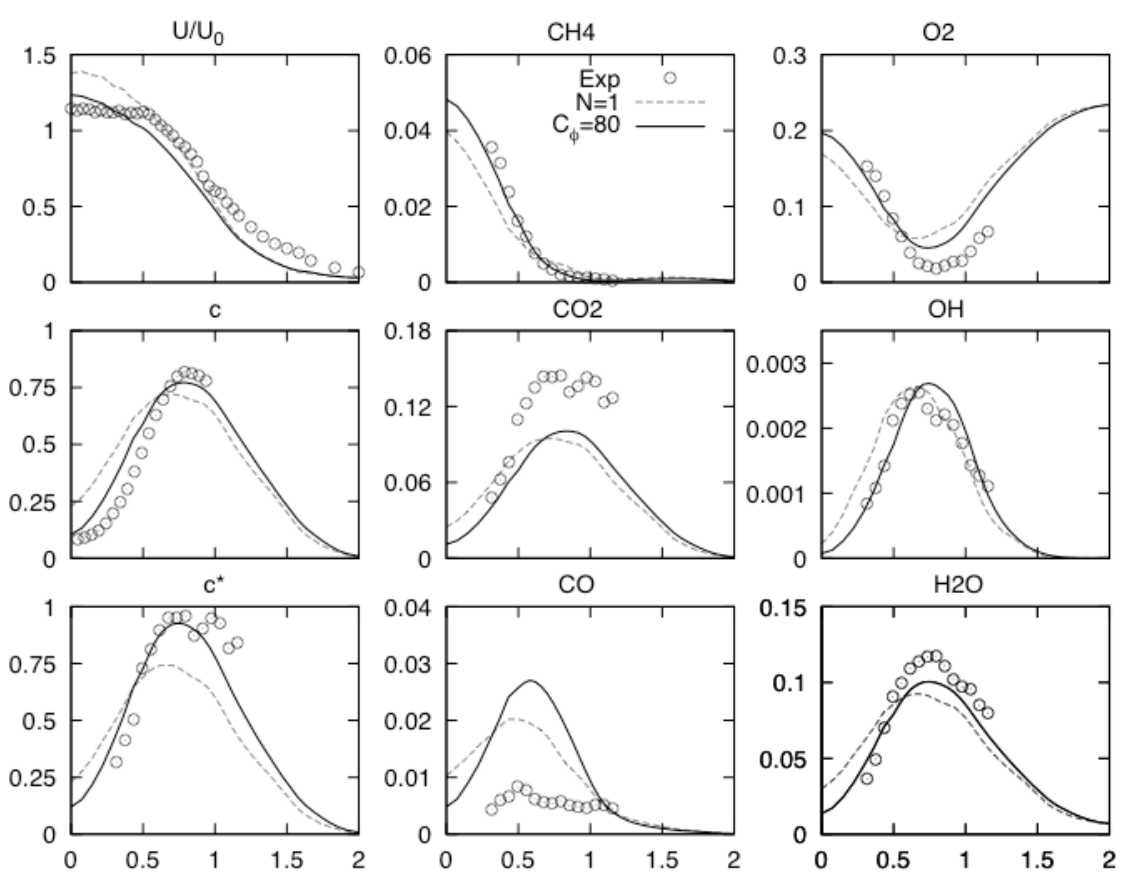

Fig. 23 Axial velocity, $U / U_{0}$, progress variable, $c$, and $\mathrm{CO}$ mass fraction distribution for the $\mathrm{F} 2$ flame with $C_{\Phi}=80$ and $N=1$

Such weakly dependence suggests that the scaling in Eq. (8) to collapse the PDF to a $\delta$ Dirac, will not play a major role. Figure 25 shows the velocity and the progress variable with the F2 flame using both scaled and non-scaled values of the micromixing constant. It can be concluded that even when using the non-scaled value of the micromixing coefficient acceptable results can be obtained (especially closer to the nozzle exit) it does not significantly affect the results, as inaccuracies in instantaneous values probably do not affect time-averaged values and its effects its limited to laminar regions. From Fig. 23 it was observed that even $C_{\Phi}=80$ gave relatively accurate and acceptable results. The largest difference can however be observed in the low Reynolds pilot/air shear layer, where no experimental data is available. 

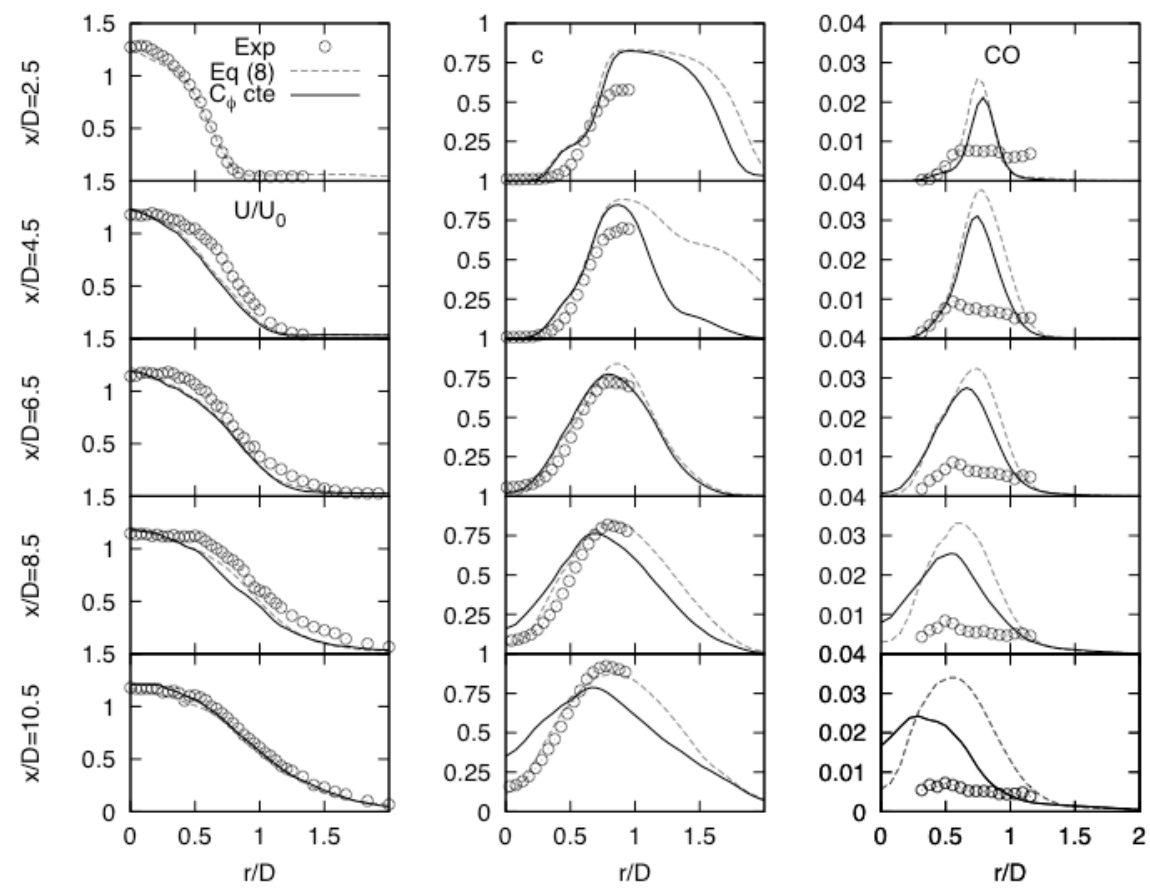

Fig. 24 Axial velocity and progress distribution for the F2 flame using Eq. (8)

\subsection{Sensitivity Analysis: Pilot Composition}

In Section 4.2-4.4, the common observation is that the $\mathrm{CO}$ mass fraction is systematically over-predicted, nearly independently of the value of the selected parameter under study. It should be noted that some values yield improved results over other values, but the general trend is that $\mathrm{CO}$ species mass fraction is always over-predicted.
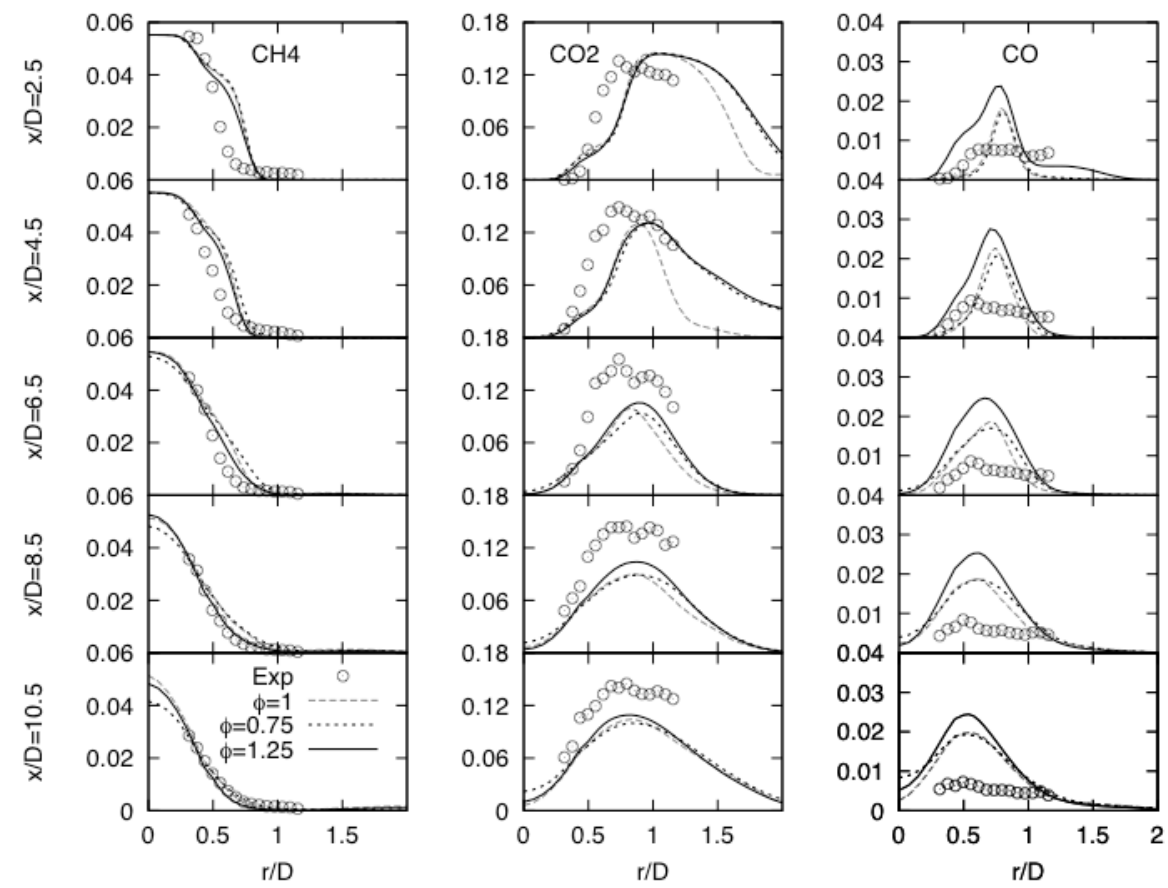

Fig. $25 \mathrm{CH}_{4}, \mathrm{CO}_{2}$ and $\mathrm{CO}$ radial distribution for the $\mathrm{F} 2$ flame with different pilot composition 
The uncertainties in the pilot composition motivated this section; three pilot compositions are examined in the F1 flame. In the original case, the pilot composition was taken as the composition of the products of combustion of a methane-air mixture at equivalence ratio $\phi=1$. Two additional cases were selected, the one with pilot composition corresponding to a lean mixture $\phi=0.75$ and with a rich one $\phi=1.25$. These values are obviously extreme and outside experimental error but they are a good indication of how pilot composition affects species prediction.

Figure 25 shows that no significant difference is observed in the prediction between the different pilot compositions at all axial stations apart from an increase of $\mathrm{CO}$ for rich pilots. Progress variable and axial velocity were unaffected (not shown).

\subsection{Sensitivity Analysis: Differential Diffusion}

In LES, molecular diffusion has a much more predominant role than in RANS and must be modelled accurately. In premixed flames, even a relatively large Reynolds number differential diffusion may play a significant role [75]. In this section the effects of nonunity Lewis numbers are investigated on the F2 flame. Full PDF solutions including differential diffusion are rare [76] and no formulation has been presented yet in the stochastic fields context (although modification in the mixing model could be directly applied [77]); therefore results with $N=1$ are presented. In Figure 26 mass fraction predictions for $\mathrm{CH}_{4}, \mathrm{CO} 2$ and $\mathrm{CO}$ are presented. The most important observation is the improvement in the prediction of $\mathrm{CO}$ and $\mathrm{CH}_{4}$ mass fraction at nearly all stations. Differential diffusion seems to affect $\mathrm{CO}$ predictions strongly by improving the shape of the average flame front. $\mathrm{CO}$ improves by about $20 \%$, especially at axial locations further away from the nozzle exit. Further improvement might be obtained if a detailed mechanism and increased $N$ were to be used.
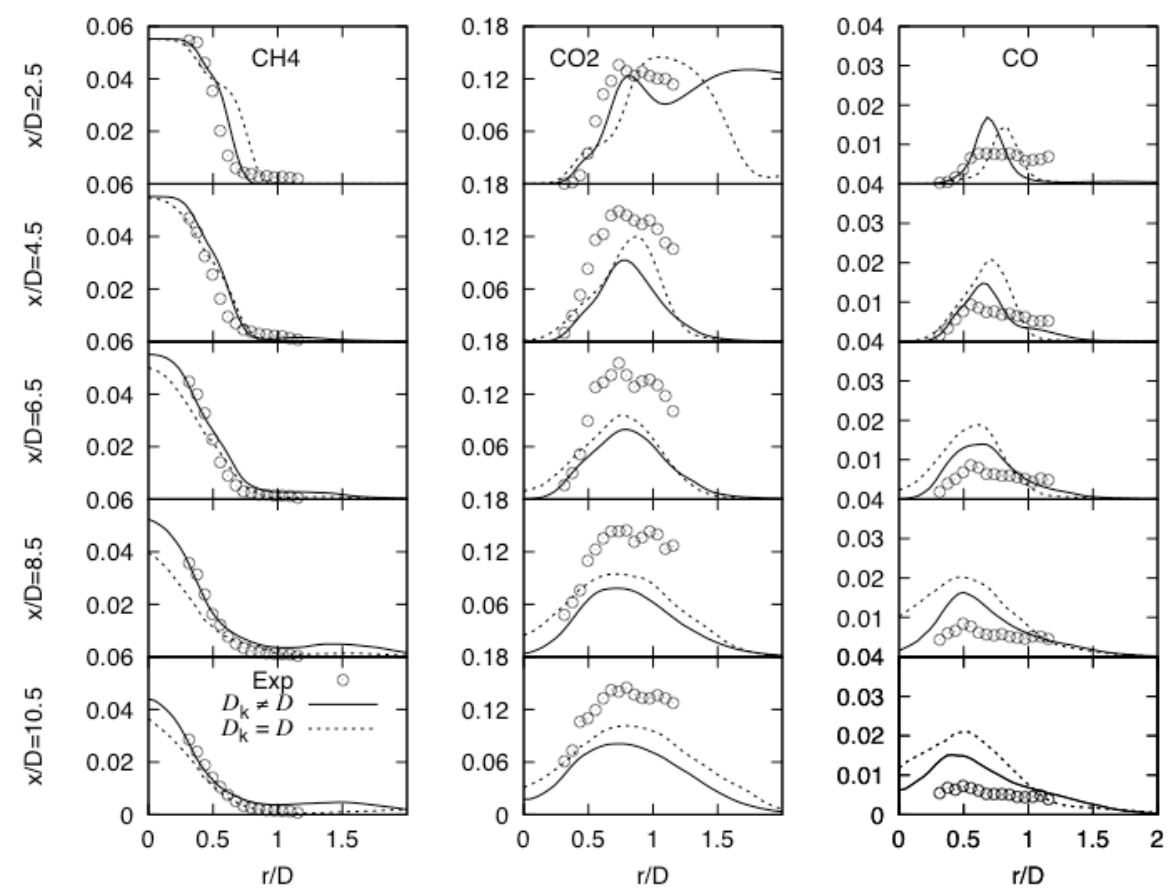

Fig. $26 \mathrm{CH}_{4}, \mathrm{CO}_{2}$ and $\mathrm{CO}$ mass fraction distribution for the $\mathrm{F} 2$ flame with and without differential diffusion with $N=1$ 


\subsection{Flame Quenching}

One of the advantages of the present LES-PDF methodology is that it can predict unsteady phenomena such as flame quenching. The solution with $N=1$ of the F1 flame was used as an initial condition for a $N=4$ field simulation in order to predict flame extinction of a hypothetical F0 flame. The axial velocity was increased gradually from 65 $\mathrm{m} / \mathrm{s}$ to $150 \mathrm{~m} / \mathrm{s}$ (in steps of $5 \mathrm{~m} / \mathrm{s}$ ). According to experimental data [54] the stoichiometric Bunsen flames begin to get extinguished at stretch rate of $S_{e} \approx 6150 \mathrm{~s}^{-1}$ where $S=U_{0} / D$ is defined as a global stretch parameter based on the exit diameter of the jet flow. From critical stretch parameter, the quenching velocity is calculated at $73.8 \mathrm{~m} / \mathrm{s}$. Numerically, quenching was observed in the region of $80-85 \mathrm{~m} / \mathrm{s}$.

Figure 29 shows the gradual decrease of the flame temperature at $0.5 \mathrm{~ms}$ intervals, while Figure 30 shows the $\mathrm{OH}$ mass fraction at the same time. The hypothetical F0 flame, progressively increase its Karlovitz number moving towards the right of the diagram in Figure 2 (right) to the distributed or broken reaction zones regime and then complete quenching. Following the discussion on the stochastic field sensitivity (section 4.3) as the simulation comes close to extinction, the influence of sub-grid modelling decreases, as there is not a defined flame structure but large scale broken reaction zones. Qualitatively the images look remarkably similar to the extinction of a non-premixed flame $[5,78]$
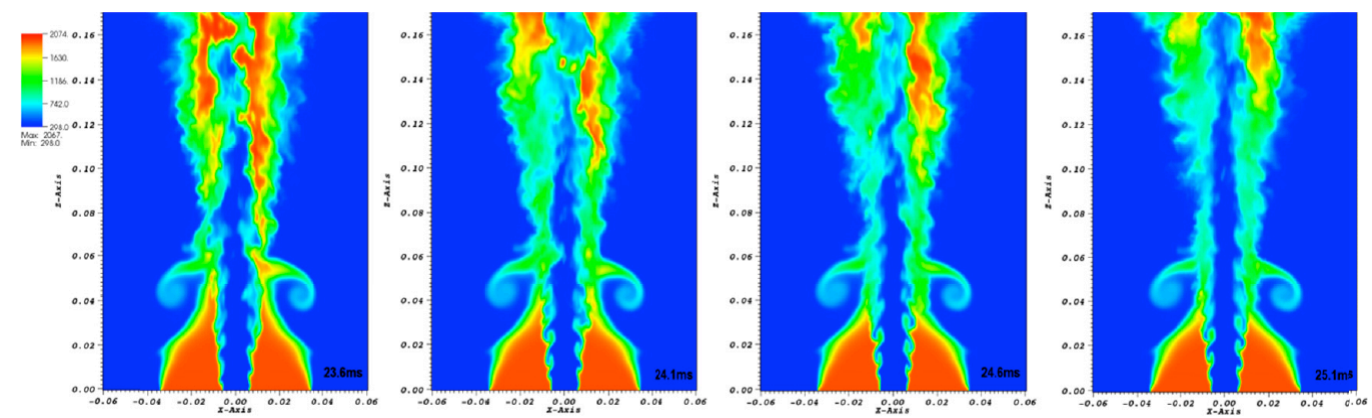

Fig. 27 Snapshots of temperature for the F0 flame at $0.5 \mathrm{~ms}$ intervals
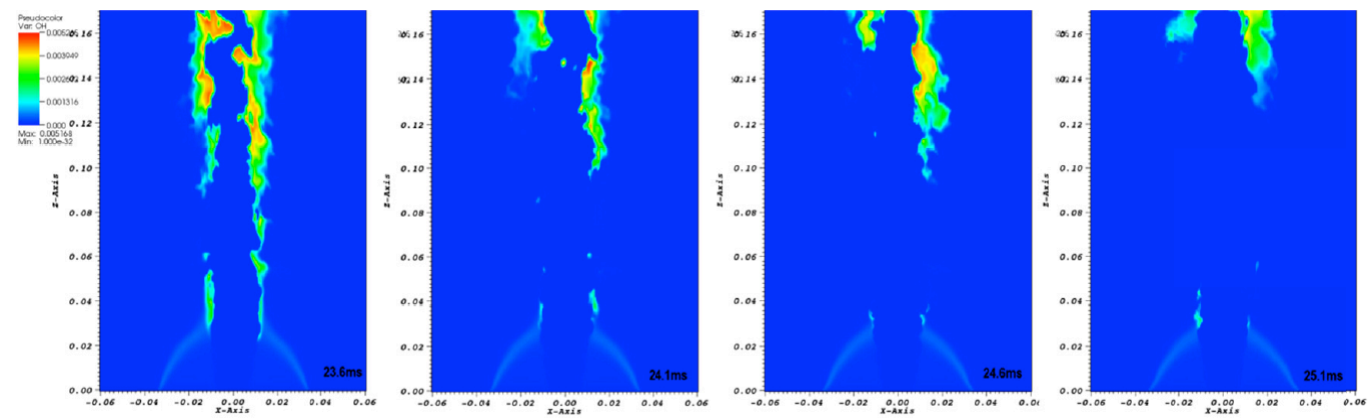

Fig. 28. Snapshots of $\mathrm{OH}$ mass fractions for the F0 flame at $0.5 \mathrm{~ms}$ intervals 


\section{Conclusions}

The paper presents one of the first applications of LES-PDF to premix flames using the stochastic fields method. The same closure models used in non-premixed flames have been used, giving comparable (if not better) results that other proposed methods over the flames studied. The LES-PDF approach is shown to work accurately with a relatively low number of fields, 16, at a range of Reynolds, 24000-52000 and Karlovitz numbers 3.4-11. The results showing little sensitivity to modeling parameters, such as the micro-mixing constant in constrast to RANS-PDF approaches in premix flames. The model is able to capture large scale quenching at qualitatively the correct extinction speed. Even in the coarse grid, the velocities, temperatures and mass fractions are captured reasonably well.

The largest Reynolds number flame, F1, is better predicted than the lower Reynolds flame, F3; where the latter is more sensitive to number of fields and sub-grid effects. The acceptable results without model $(N=1)$ suggest that the sub-grid effects are limited and not so important at large Karlovitz numbers. This is agreement with previous findings [69] where neglecting sub-grid combustion modelling (ILES approach) provide good agreement on a high Karlovitz 1000 premix flame. However at low Karlovitz numbers, in the thin reaction regime, sub-grid combustion modelling plays a larger role. This analysis is consistent as long as the filter width is maintained constant, which implies that as the Karlovitz number increases, the regimes move away from the line $D a_{\Delta}=1$ (See Fig 2) and therefore sub-grid effects are limited.

Nevertheless, the calculations show large CO over-predictions at all flames, which cannot be explained by the chemical mechanisms or the pilot composition. Similar discrepancies are reported in the literature $[24,25,55]$. A progress variable based on $\mathrm{CO}$ and $\mathrm{CO}_{2}$ shows good agreement with experimental data, suggesting that the error may be in the $\mathrm{CO}$ concentration in the pilot.

Finally, the inclusion of differential diffusion effects generally leads to small improvements of mass fractions predictions, albeit larger than switching to more detailed chemistry. Unfortunately no simple formulation exists for differential diffusion for the stochastic fields method and the full benefit of including it is not clear.

\section{References}

1. Mustata, R., Valiño, L., Jimenez, C., Jones, W. P., Bondi, S.: A probability density function Eulerian Monte Carlo field method for Large eddy simulations: Application to a turbulent piloted methane/air diffusion flame (Sandia D). Combust. Flame 145, 88-104 (2006).

2. Jones, W. P., Navarro-Martinez, S.: Large Eddy Simulation of auto-ignition with a subgrid probability density function method. Combust. Flame 150 (3), 170-187, (2007).

3. Jones, W.P., Navarro-Martinez, S., Röhl, O.: Large Eddy simulation of hydrogen autoignition with a probability density function method. Proc. Combust. Inst. 31 1765-1771, (2007).

4. Raman V., Pitsch H., A consistent LES/filtered-density function formulation for the simulation of turbulent flames with detailed chemistry, Proc. Combust. Inst. 31(2), 17111719, (2007).

5. Jones, W.P., Prasad N., Large Eddy Simulation of the Sandia Flame Series (D-F) using the Eulerian stochastic field method', Combust. Flame 157, 1621-1636, (2010).

6. Peters N., Multiscale Combustion and Turbulence, Proc. Combust. Inst. 32, 1-25, (2009). 
7. Poinsot, T., Veynante, D., Theoretical and Numerical Combustion, Second Edition, R. T. Edwards, (2005).

8. Fureby, C., Löfström, C.: Large Eddy Simulations of bluff body stabilized flames Proc. Combust. Inst. 25, 1257-1264, (1994)

9. Colin, O., Ducros, F., Veynante, D. and Poinsot, T.: A thickened flame model for large eddy simulations of turbulent premixed combustion. Phys. Fluids, 12(7), 1843-1863 (2000)

10. Im, H. G., Lund, T. S. and Ferziger, J. H.: Large Eddy Simulation of turbulent front propagation with dynamic subgrid models. Phys. Fluids, 9(7), 3826-3833 (1997)

11. Pitsch, H., de Lageneste L. D.: Large Eddy Simulation of Premixed Turbulent Combustion using a Level-Set Approach, Proc. Combust. Inst, 29, 2001-2008, (2002)

12. Boger, M., Veynante, D., Boughanem, H., Trouve, A.: Direct Numerical Simulation analysis of flame surface density concept for large eddy simulation of turbulent premixed combustion. Proc. Combust. Inst. 27, 917-927, (1998)

13. Hawkes E R, Cant R.S: A flame surface density approach to large-eddy simulation of premixed turbulent combustion, Proc. Combust. Inst. 28, 51-58, (2000)

14. Weller, H. G.,Tabor, G., Gosman, A. D., Fureby, C.: Application of a flame-wrinkling LES combustion model to a turbulent mixing layer. Proc. Combust. Inst. 27, 899-907, (1998)

15. Richard, S., Colin, O., Vermorel, O., Benkenida, A., Angelberger, C., Veynante, D: Towards large eddy simulation of combustion in spark ignition engines, Proc. Combust. Inst. 31, 3059-3066, (2007)

16. van Oijen, J. A., de Goey, L.P.H.: Modelling of Premixed Counterflow Flames using the Flamelet-Generated Manifold Method, Combust. Theor. Model, 6(3), 463-478, (2002).

17. Knikker, R., Veynante, D., Menevau, C.: A priori testing of a similarity model for large eddy simulations of turbulent premixed combustion. Proc. Combust. Inst. 29, 2105-211, (2002)

18. Kim, W-W., Menon, S.: Numerical Modeling of Turbulent Premixed Flames in the ThinReaction-Zones Regimes. Combust. Sci. Tech, 160, 119-150 (2000)

19. Pitsch, H.: Large Eddy Simulation of Turbulent Combustion, Annu. Rev. Fluid Mech., 38, 453-482 (2006)

20. Chakravarthy, V. K., Menon, S.: Large Eddy Simulation of Turbulent Premixed Flames in the flamelet regime, Combust. Sci. Tech., 162, Pages 175-190, (2001).

21. Lecocq, G.,Richard, S., Colin, O., Vervisch, L.: Hybrid presumed pdf and flame surface density approaches for Large-Eddy Simulation of premixed turbulent combustion, Combust. Flame 158, 1201-1214, (2011).

22. Hernández-Pérez, F.E., Yuen, F. T. C., Groth, C. P. T., Gülder, Ö.L.: LES of a laboratoryscale turbulent premixed Bunsen flame using FSD, PCM-FPI and thickened flame models, Proc. Combust. Inst. 33, 1365-1371, (2011)

23. Kuenne, G., Ketelhuen, A., Janicka, J.:LES modelling of premixed combustion using a thickened flame approach coupled with FGM tabulated chemistry. Combust. Flame 158, 1750-1767, (2011).

24. Stöllinger, M., Heinz, S.: PDF Modelling and simulation of premixed turbulent combustion, Monte Carlo Method. Appl. 14 (4), 343-377 (2008)

25. Lindstedt, R. P., Vaos, E. M.: Transported PDF modelling of high-Reynolds-numbre premixed turbulent flames, Combust. Flame, 145 495-511 (2006).

26. Lindstedt, R. P., Milosavljevic, V. D., Persson, M.: Turbulent burning velocity predictions using transported PDF methods, Proc. Combust. Inst. 33, 1277-1284, (2010)

27. Rowinski, D. H., Pope S.B., PDF calculations of piloted premixed jet flames, Combust. Theor. Model 15(2), 245-266 (2011).

28. Stöllinger, M K., Stefan, H.: Evaluation of scalar mixing and time scale models in PDF simulations of a turbulent premixed flame, Combust. Flame 157, (9), (2010). 
29. Cannon, S. M., Brewster, B.S., Smoot, L. D.: PDF modeling of lean premixed combustion using in situ tabulated chemistry, Combust. Flame 119, (3), 233-252 (1999)

30. Jones, W.P., Navarro-Martinez, S.: Large Eddy Simulation of a premixed turbulent flame using a Probability Density Function Approach: In Proceedings of the European Combustion Meeting, Cardiff, Wales (2011).

31. Yilmaz, S.L., Nik, M.B., Givi, P., Strakey P.A.: Scalar Filtered Density Function for Large Eddy Simulation of a Bunsen Burner, Journal Propulsion and Power, 26(1), 84-93, (2010)

32. Kempf A.: Large Eddy Simulation of Non-Premixed Turbulent Flames, Ph.D. Thesis, Technischen Universitat Darmstadt (2003).

33. di Mare, F.: Large Eddy Simulation of Reacting and non-Reacting Turbulent Flows, Ph.D. Thesis, Imperial College London (2002)

34. Gao, F., O'Brien, E.: A large eddy simulation scheme for turbulent reacting flows. Phys Fluids, 9(7), 1282-1284 (1993)

35. Schmidt, H., Schumann, U.: Coherent structure of the convective boundary layer derived from large-eddy simulations. J Fluid Mech., 200, 511-562 (1989)

36. Jones, W.P., Navarro-Martinez, S.: Large Eddy Simulations and the Filtered Probability Density Function Method, LES and DNS of Ignition and Complex-Structure Flames with Local Extinction, AIP Proceedings (2009).

37. Branley, N., Jones, W.P.: Large Eddy Simulation of a turbulent non-premixed flame, Combust. Flame, 127(1), 1914-1934, (2001).

38. Dopazo, C., O’ Brien, E.: Functional formulation of non isothermal turbulent reactive flows. Phys. Fluids, 17 (1974) 1968.

39. Villermaux, J., Devillon, J. C.: Représentation de la coalescence et de la redispersion des domaines de ségrégation dans un fluide par un modèle d'interaction phénoménologique, Proceedings of the 2nd International Symposium on chemical reaction engineering, (1972).

40. Fox R.O.: Computational Models for Turbulent Reacting Flows, Cambridge University Press, 2003.

41. Haworth, D.C.: Progress in probability density function methods for turbulent reacting flows, Prog. Ener. Combust. Sci. 36 (2) 168-259 (2010).

42. Prasad, N.: Large Eddy Simulation of Partially Premixed Turbulent Combustion, Ph.D. Thesis, Imperial College London, (2011).

43. Warhaft, Z., Lumley, J.L.: Experimental Study of Decay of Temperature-Fluctuations in Grid-Generated Turbulence, J Fluid Mech., 88, 659-684 (1978)

44. Kulkarni, R., Polifke, W.: LES of Delft-Jet-In-Hot-Coflow (DHJC) wth Tabulated Chemistry and Stochastic Fields Combustion Model, $11^{\text {th }}$ International Conference on Combustion and Energy Utilization, Coimbra, Portugal 2012

45. Jones, W. P., Prasad, V N: LES-pdf simulation of a spark ignited turbulent methane jet. Proc. Combust. Inst. 33, 2153-2160 (2011)

46. Jones, W. P., Lyra, S, Navarro-Martinez, S.: Large Eddy Simulation of a swirl stabilized spray flame, Proc. Combust. Inst. 33, 1355-1363 (2011)

47. Jones, W. P., Lyra, S, Navarro-Martinez, S.: Numerical investigation of swirling kerosene spray flames using Large Eddy Simulation, Combust. Flame. 33, 1539-1561 (2012)

48. Valiño, L: A Field Monte Carlo Formulation for Calculating the Probability Density Function of a Single Scalar in A Turbulent Flow. Flow, Turb. Combust 60, 157-172 (1998)

49. Sabel'nikov, V, Soulard, O: White in Time Scalar Advection Model as Tool for Solving Joint Composition PDF equations. . Flow, Turb. Combust 77, 333-357 (2006)

50. Soulard, O: Approaches PDF pour la combustion turbulente, Ph.D. Thesis, Universite de Rouen (2005).

51. Jones W.P., di Mare F., Marquis A.J.: LES BOFFIN: Users Guide, Technical Memorandum, Imperial College London, 2002. 
52. Gardiner, C: Handbook of Stochastic methods, Springer-Verlag, New-York (1985)

53. Kloeden, P. E., Platen, E: Numerical Solution of stochastic differential equations, Springer-Verlag, New-York (1992)

54. Chen, Y.C., Peters, N., Schneemann, G.A., Wruck, N., Renz ,U., Mansour, M. :The detailed Flame Structure of Highly Stretched Turbulent Premixed Methane-Air Flames. Combust. Flame 107,223-244 (1996).

55. Herrmann, M.: Numerical simulation of turbulent Bunsen flames with the level set flamelet model, Combust. Flame, 145,357-375, (2006).

56. Salehi, M; Bushe, W.: presumed PDF modelling for RANS simulation of turbulent premixed flames, Comb. Theor. Model. 14, 381-403 (2010)

57. Stöllinger, M K., Stefan H.: Evaluation of scalar mixing and time scale models in PDF simulations of a turbulent premixed flame, Combust. Flame 157, (9), 1671-1685 (2010).

58. Kolla, H.: Scalar Dissipation Rate based Flamelet Modelling of Turbulent Premixed Flames', Ph.D. Thesis, University of Cambridge (2009).

59. Salehi, M., Bushe, K., Daun, K.: Application of the conditional source term estimation for turbulence chemistry interactions in a premixed flame. Combust. Theor. Model 16(2),301-320 (2012)

60. Prasad, R. O. S., Gore, J. P.: An Evaluation of Flame Surface Density Models for Turbulent Premixed Jet Flames, Combust. Flame 116, 1-14 (199)

61. Peters, N; Turbulent Combustion, Cambridge University Press, (2000)

62. Pitsch H., Steiner H.: Large Eddy simulation of a turbulent piloted methane/air diffusion flame (Sandia Flame D), Phys. Fluids, 12,(10), 2541-2554, (2010).

63. Akselvoll, K., Moin, P.: Large-eddy simulation of turbulent confined coannular jets. J. Fluid Mech 315, 387-411, (1996).

64. Danaila, I. and Boersma, B.J.: Direct Numerical Simulation of bifurcating Jets, Phys. Fluids 12(5), 1256-1257 (2000).

65. Navarro-Martinez S., Kronenburg A., di Mare, F.: Conditional Moment Closure for Large Eddy Simulations, Flow, Turb. Combust. 75, 245-274 (2005)

66. Sung, C.J., Law C.K. and Chen, J.Y. : Augmented Reduced mechanisms for NO emission in methane oxidation, . Combust. Flame, 125, 906-919, (2001).

67. Jones, W.P., Lindstedt, R. P.: Global reaction schemes for hydrocarbon combustion, Combust. Flame, 73, (3), 233-249 (1988)

68. Kuo, K K: Principles of Combustion, John Wiley \& Sons, New Jersey $2^{\text {nd }}$ Edition (2005)

69. Duwig, C., Nogenmyr, K. J., Chan, C., Dunn M.J.: Large Eddy Simulations of a piloted lean premix jet flame using finite rate chemistry, Combust. Theor. Model. 15 (4), 537568, (2011).

70. Duwig, C, Fuchs, L: Large eddy simulation of a H2/N2 lifted flame in a vitiated co-flow, Combust. Sci. Technol. 180, 453-480 (2008)

71. Fureby, C: Comparison of flamelet and finite rate chemistry LES for premixed turbulent combustion, AIAA Paper 2007-1413 (2007)

72. Pope, S.B.: Monte Carlo calculations of premixed turbulent flames, Proc. Combust. Inst, 18,1001-1010 1981.

73. Overholt, M.R., Pope, S.B.: Direct Numerical Simulation of a passive scalar with imposed mean gradient in isotropic turbulence, Phys. Fluids, 8, (11), 3128-3148, (1996).

74. Rowinski, D, Pope, S. B.: PDF calculations of piloted premixed jet flames. Combust. Theor. Model 15(2),245-266 (2011)

75. Chakraborty, N., Cant, R. S.: Influence of Lewis number on curvature effects in turbulent premixed flame propagation in the thin reaction zones regime, Phys. Fluids 17, 105105 (2005)

76. McDermott, Pope, S. B.: A particle formulation for treating differential diffusion in filtered density function methods, H. Comp. Phys 226 (1) 947-993 (2007)

77. Richardson, E. S., Chen, J. H.: Application of PDF mixing models to premixed flames with differential diffusion. Combust. Flame, 159, 2398-2414 (2012) 
78. Garmory, A and Mastorakos, E: Capturing localised extinction in Sandia Flame F with LES-CMC, Proc. Combust. Inst., 33. 1673-1680, (2011)

79. Jones, W P, Marquis, A J and Prasad, V N. Les of a turbulent premixed swirl burner using the eulerian stochastic field method. Combust. Flame, 159(10), 3079-3095 (2012)

80. Aspden, A J, Day, M S and Bell, J B, Turbulence-flame interactions in lean premixed hydrogen: transition to distributed burning regime, J Fluid. Mech., 680, 287-320 (2011)

81. Aspden, A J. Bell, J B, Woosley, S E, Distributed Flames in Type Ia supernovae, The Astrophysical Journal 710(2) 1654-1663 (2010)

82. Wang, G, Boileau, M and Veynante, D, Combust. Flame, Implementation of a dynamic thickened flame model for large eddy simulations of turbulent premixed combustion 158(11), 2199-2213 (2011)

83. Jones, W P and Tyliszczak, A, Large Eddy Simulation of Spark Ignition in a Gas Turbine Combustor, Flow, Turb. Combust 85, 711-734 (2010) 